\title{
Scalar democracy
}

\author{
Christopher T. Hill, ${ }^{1, *}$ Pedro A. N. Machado, ${ }^{1, \dagger}$ Anders E. Thomsen, ${ }^{2, \$}$ and Jessica Turner ${ }^{1, \S}$ \\ ${ }^{1}$ Fermi National Accelerator Laboratory, P.O. Box 500, Batavia, Illinois 60510, USA \\ ${ }^{2} C P^{3}$-Origins, University of Southern Denmark, Campusvej 55, DK-5230 Odense M, Denmark
}

(Received 25 February 2019; published 15 July 2019)

\begin{abstract}
We conjecture that there exists a scalar bound state for every pair of fundamental fermions at a UV ("composite") scale, $\Lambda \gg v_{\text {weak }}$. This implies a large number of universally coupled, subcritical Higgs doublets. All but the standard model Higgs are "dormant," with large positive squared masses, and each receives a small vacuum expectation value via mixing with the standard model Higgs. Universal couplings, modulo renormalization group running effects, flip the flavor problem into the masses and mixings of the Higgs system. Doublets associated with heavy fermion masses, $b, c, \tau$, likely lie in the multi-tera electron volt range but may be observable at the current LHC, or a high-luminosity and/or an energy-upgraded LHC. In the lepton sector, we are led to a Higgs seesaw for neutrino masses and corollary processes of observable flavor violation. The observation of the first sequential doublet coupled to $\bar{b} b$ with mass less than $3.5 \mathrm{TeV}$ would lend credence to the hypothesis.
\end{abstract}

DOI: 10.1103/PhysRevD.100.015015

\section{INTRODUCTION}

In the present paper, we propose that every standard model fermion pair is associated with a complex scalar boson, perhaps due to binding by a universal attractive interaction at a very high scale, $\Lambda$. Among many new states, including leptoquarks, colored isodoublets and singlets, etc., this hypothesis implies the existence of a large number of Higgs bosons. One of the main features of this is the approximate equality of all scalar-fermion, and, in particular, Higgs-Yukawa, couplings, modulo renormalization group effects.

We assume the lightest of these is the standard model Brout-Englert-Higgs boson (SMH). The remaining doublets are sequentially heavier with positive $M^{2}$ 's, i.e., "dormant." They will have universal couplings to their constituent fermions at $\Lambda$ but renormalized couplings at the electroweak scale that are $g \simeq 1$ for quarks and $g_{\ell} \simeq 0.7$ for leptons. Each standard model (SM) fermion acquires its mass through its coupling to the particular Higgs doublet that is comprised of said fermion, which in turn mixes with

\footnotetext{
*hill@fnal.gov

†pmachado@fnal.gov

*aethomsen@cp3.sdu.dk

§iturner@fnal.gov

"We use the term "dormant" as distinct from "inert," which to us implies electroweak sterile scalars.
}

Published by the American Physical Society under the terms of the Creative Commons Attribution 4.0 International license. Further distribution of this work must maintain attribution to the author(s) and the published article's title, journal citation, and DOI. Funded by SCOAP ${ }^{3}$. the SMH to acquire a perturbative "tadpole" mass. In particular, our present model is "subcritical": the negative $M^{2}$ of the SMH arises from mixing with dormant Higgses. The associated Higgs bosons thus become lighter for the heavier fermions and very heavy for the neutrinos. The spectrum of masses and mixings among these heavy scalars is the origin of flavor physics.

Though we have no theory of the masses and mixings of the large array of composite scalars, we can make use of phenomenology. The model then becomes predictive, essentially because the Yukawa couplings are determined. We call this system "scalar democracy" as it bears some remote similarity to the "nuclear democracy" of the late 1960s.

Sequential Higgs bosons have certainly been considered previously, and we cannot review the vast literature. Nonetheless, few theorists venture beyond a few Higgs bosons. Many Higgs bosons arise in the context of an extended gravity, such as a scheme described to us by Bjorken [1], which inspired our thinking about many Higgs scalars. Our key new ingredient here is the idea of approximate Higgs-Yukawa coupling constant universality. Some relevant models that presage our present discussion are Refs. [2-4]. A Coleman-Weinberg mechanism and Higgs portal interactions led one of us to a second Higgs doublet coupled to $\bar{b} b$ with $g=1$ at about $400 \mathrm{GeV}$ (see Ref. [5] and a long list of references therein). In our present model, the SMH is a $\bar{t} t$ composite state [6-8], but the predictions of minimal composite $\bar{t} t$ are significantly modified by mixing. We note that recent work attempting to construct an "asymptotically safe" UV completion of the SM arrives at a structure in the Higgs 
sector similar to ours [9-11], but for different reasons. The phenomenology of many Higgs bosons is, to our knowledge, essentially unexplored. In a companion paper, we give further details in a truncated version applied to the third generation [12].

In our particular scenario, we count 18 sequential colorless doublets in the quark sector and 18 in the lepton sector. In the quark sector, this includes the lightest doublet, $H_{0}$, associated with the top quark, $\left(\bar{t}_{\mathrm{L}}, \bar{b}_{\mathrm{L}}\right) \tilde{H}_{0} t_{\mathrm{R}}$ (where $\tilde{H}_{0}$ is the charge conjugated Higgs), which is identified with the SM Higgs doublet. This establishes the universal quarksector Yukawa coupling to be $g=1$. The quark masses and mixings are then determined by the spectrum of the 18 quark-sector Higgs doublets. We will see the Higgs doublet associated with the $b$ quark, $g\left(\bar{t}_{\mathrm{L}}, \bar{b}_{\mathrm{L}}\right) H_{b} b_{\mathrm{R}}$, is expected to have a mass of approximately 3 to $5 \mathrm{TeV}$, though in some limit, it could be fortuitously lighter. We also have 18 doublets in the charged lepton sector. With the possible exception of $H_{\tau}$, these tend to be much heavier as lepton masses are small. This framework provides three alternatives for the neutrino mass generation. Interestingly, neutrinos could be Dirac or Majorana via a type-I or -II seesaw mechanism. Many of the scalars providing quark and lepton masses are well beyond present collider reach. The lighter ones, associated with the $b$ and $c$ quarks, as well as the $\tau$ lepton, may have thresholds in the sub-10 $\mathrm{TeV}$ range and thus may be accessible to the LHC and future upgrades and higher-energy machines [12]. However, the heavier states may leave indirect imprints on flavor-changing observables in the quark and lepton sectors.

Scalar democracy for us is new dynamics with subcritical compositeness due to a universal interaction in the far UV. We thus blend a few key ideas from compositeness and mainly emphasize that the Yukawa couplings are universal at $\Lambda$, subject only to renormalization group evolution from $\Lambda$ to the weak scale. This, together with the input masses and mixings of the Higgs bosons, flips the quark and lepton-flavor problems away from the issue of understanding a fundamental Yukawa coupling matrix. The puzzle of fermion mass hierarchy becomes one of understanding and disentangling the multi-Higgs mass spectrum. This conversion of the flavor problem is an interesting exercise in its own right, one of which we only scratch the surface.

It should be emphasized that it is generally hard to understand the small Yukawa couplings in the SM, such as $g_{\text {electron }} \sim 10^{-6}$. These cannot be generated perturbatively from zero, owing to custodial chiral symmetries. It is natural that $g_{\text {electron }} \sim 1$ in its coupling to a new Higgs $H_{e}$, but through mass mixing, the induced coupling to the $\mathrm{SMH}$ becomes small. This is a key motivation for a scheme such as the one presented here: the small $g$ 's start out as $g \sim 1$ but are then power-law suppressed through mixing; the only ingredients we consider for this are in the extended Higgs sector with universal couplings.
As we have emphasized, the present scheme does not provide any explanation of the multiple Higgs masses and mixings. We largely depart from many of the conventional ideas in vogue, such as supersymmetry and extra dimensions. We will not concern ourselves with the overall naturalness issues, and we treat symmetry-breaking effects as inherent in the Higgs mass terms. This is analogous to "soft symmetry breaking" in chiral Lagrangians and resonance models of the 1960s. We do impose fine-tuning constraints in the low-energy effective theory of $H_{b}$, in which the mixing generates the largest feedback on the SMH, $H_{0}$ (level repulsion), and the negative $M^{2}$ of the SMH can arise from this effect (for a more detailed recent discussion of this issue, see Ref. [12]).

It is our conclusion, at least at a first pass with various simplifying assumptions made along the way, that such a theory can exist. New phenomena may show up at a high luminosity and/or energy-doubled LHC, and certainly at a $100 \mathrm{TeV}$ collider. $H_{b}$, in its lowest mass limit of approximately $1 \mathrm{TeV}$, is already accessible to the LHC, while it would be seen in the higher mass range of approximately $3 \mathrm{TeV}$ in upgraded LHC runs. In our opinion, a robust theoretical spectroscopy of multiple Higgs bosons offers a rationale for luminosity and energy upgrades of the LHC and future ultraenergetic machines. If the upgraded LHC were to fail to discover a pair of isodoublet Higgs bosons with universal coupling, such as the lightest $H_{b}$ or $H_{\tau}$ states in our model, then this scheme would be disfavored.

Analyzing, at least schematically, the phenomenological consequences and constraints of the scalar democracy hypothesis is our main goal. We begin in Sec. II with a theoretical "motivation" for this perspective. Then, in Sec. III, we summarize the dynamics of our model at low energies and arrive at a fairly simple effective Lagrangian describing the couplings to quarks and leptons of the multiHiggs spectrum. This is followed by a more detailed discussion of phenomenology in the subsequent section, Sec. IV. Here, we discuss the main production channels and collider prospects for the dormant Higgses. We will also discuss the implications of the dormant Higgses on flavor physics and the resulting bounds on their masses.

A reader interested in a summary of the observable features of the model, including neutrino masses, may skip directly to Sec. IV.

\section{THEORETICAL MOTIVATION}

\section{A. Are there many scalars in nature?}

Gravity is a universal attractive interaction. All pairs of fundamental fermions must have attractive gravitational scattering amplitudes. Near the Planck scale, this may involve exotic, new strong dynamics, new condensates, instantons, etc., and perhaps a new way to generate hierarchies.

For example, enhanced gravitational interactions may trigger condensates, dynamically generating Majorana 
masses for the right-handed neutrinos [13]. Similar effects may arise through gravitational instantons [14]. Alternatively, intriguing extensions of gravity, such as braneworld models, extra dimensions [15], or "bigravity" $[16,17]$, have ingredients that may likewise produce universal attractive interactions at various scales. We can also generate a large subset of these scalars by postulating new, strong gauge dynamics, which is more concrete but will not be developed presently.

We assume that a universal attractive interaction generates bound-state scalar fields at a high-energy scale $\Lambda$ (which may be of order $M_{\text {Planck }}$, but could be lower). Our hypothesis is general and transcends a wide class of possible models. While we invoke a universal pairing force, such as gravity, this is nonetheless a schematic proposal. However, when we tie this to the SMH, it becomes predictive.

We suppose the new scalar bosons are field-theoretic bound states of pairs of SM fermions. In analogy to the Nambu-Jona-Lasinio (NJL) model [18], the constituent fermions are free (unconfined), and any fermion pair couples with a common Yukawa coupling $g$ at scale $\Lambda$ to its bound-state scalar. We thus view the dynamics of composite Higgs scalars in the original framework [6-8], mainly devolving along the lines discussed by Bardeen et al. [7]. In this, we have a factorization of the interaction into auxiliary fields at scale $\Lambda$, and we then flow into the infrared by the renormalization group (RG), whereby the auxiliary fields become dynamical. This implies that the renormalized Higgs-Yukawa (HY) couplings are given by Landau poles at the scale $\Lambda$, and we remark below that the extended Higgs sector then pulls the top HY coupling into concordance with experiment, $g_{\text {top }} \approx 1$. So, rather than starting with the NJL four-fermion interaction, here we will simply postulate the factorization of the interaction into auxiliaries, and we will not give a detailed discussion of the RG treatment. Note that this is a field-theoretic relativistic binding, and the usual nonrelativistic intuition of bound states does not apply [7]. The AdS/CFT approach to compositeness, which came later, would be interesting to explore in this context, but we expect the usual results and intuition of Ref. [7] to hold.

If the new interaction is medium strong (subcritical), then bound-state scalars will form with masses which will be below $\Lambda$ and positive. The formation of these bound states does not break any symmetries since a composite scalar inherits the quantum numbers of its constituents. Hence, the scalars, in the absence of mixing effects, are presumably degenerate with mass $M^{2}<\Lambda^{2}$ and cannot break flavor or gauge symmetries, which would trigger proton decay, etc. We do not have a detailed theory of the scalar mass spectrum, in particular, and do not provide a fine-tuning mechanism to generate a large hierarchy. Moreover, we require symmetry-breaking and mixing effects to further empower the scenario. All of these effects will be considered to be soft symmetry-breaking or "relevant" operators and will be simply inserted by hand. While the dynamics determining the spectrum is unknown, we know that it must respect the SM gauge symmetries. Rather than trying to concoct a theory of masses and mixing angles among a large number of Higgs doublets over a large range of scales, we will assume such a theory exists and let phenomenology guide us.

We thus connect the hypothesis of a rich composite Higgs spectrum with the SM by postulating that the SMH is the lightest scalar doublet. This would then be the $\left(\bar{t}_{\mathrm{L}}, \bar{b}_{\mathrm{L}}\right) t_{\mathrm{R}}$ bound-state element of the scalar complex $[6,7]$, and, indeed, the SMH is a $\bar{t} t$ bound state as in the original composite Higgs models [6-8]. The top-quark Yukawa coupling, $g$, is then the universal coupling for all quark pairs to their particular Higgs doublets. Leptons will likewise have a universal coupling to their Higgs bosons, $g_{\ell}$, but we expect that the RG running will yield a ratio $g_{\ell} / g \sim 0.7$, due mainly to QCD (this is the analogue of the $\mathrm{SU}(5)$ relation for $m_{b} / m_{\tau}$ [19]). In fact, $g=1$ is close to the RG fixed point for the top quark [20,21], which is not far from the prediction of compositeness [7], and in fact this scheme can bring the RG fixed point into concordance with $m_{t}=173 \mathrm{GeV}$ [see Eq. (16) and the discussion below].

\section{B. Sketch of the dynamics}

The dynamics of the lowest-lying sequential Higgs bosons is rather simple. Let us anticipate the dynamical implications in the case of the top and bottom quarks and the three generations of neutrino masses (see also Ref. [12]).

The $\mathrm{SMH}, H_{0}$, in isolation has the usual potential

$$
V_{\mathrm{Higgs}}=-M_{0}^{2} H_{0}^{\dagger} H_{0}+\frac{\lambda}{2}\left(H_{0}^{\dagger} H_{0}\right)^{2},
$$

where $-M_{0}^{2}$ is negative, and phenomenologically $M_{0} \simeq$ $88.4 \mathrm{GeV}, \lambda \simeq 1 / 4$ (the observed physical Higgs boson mass is $\sqrt{2} M_{0} \simeq 125 \mathrm{GeV}$ ).

New sequential Higgs doublets, $H_{x}$, are dormant, meaning they have the usual SMH electroweak quantum numbers, but owing to large, positive mass terms, $M_{x}^{2} H_{x}^{\dagger} H_{x}$, they do not directly undergo condensation. However, in order to generate the light-quark and lepton masses, they must have small mixings to the SMH,

$$
\begin{aligned}
V= & M_{H}^{2} H_{0}^{\dagger} H_{0}+\frac{\lambda}{2}\left(H_{0}^{\dagger} H_{0}\right)^{2} \\
& +\sum_{x}\left(M_{x}^{2} H_{x}^{\dagger} H_{x}-\mu_{x}^{2} H_{0}^{\dagger} H_{x}+\text { H.c. }\right),
\end{aligned}
$$

parametrized by $\mu_{x}$. The vacuum expectation values (VEVs) of the dormant Higgses are small, so their quartic interaction terms should generally be negligible, and we ignore them. 
The mass mixing causes each $H_{x}$ to acquire a small VEV (tadpole) of order

$$
\left\langle H_{x}\right\rangle=\left(\begin{array}{c}
0 \\
\mu_{x}^{2} v / M_{x}^{2}
\end{array}\right),
$$

where $v \simeq 174 \mathrm{GeV}$ is the electroweak VEV.

Because of the mixing, $H_{0}$ is then "level repelled" down by an amount or order $\mu_{x}^{4} / M_{x}^{2}$ :

$$
-M_{0}^{2}=M_{H}^{2}-\sum_{x} \frac{\left|\mu_{x}^{2}\right|^{2}}{M_{x}^{2}} .
$$

An implementation of this mechanism in supersymmetric models appears in Ref. [22] and in a simple left-right symmetric scheme in Ref. [12]. Therefore, starting from a positive mass term, $M_{H}^{2}$, in Eq. (2), this cumulative effect can explain why the SMH boson has the tachyonic, or negative, $-M_{0}^{2}$, in Eq. (1).

Hence, there is only a single condensate associated with a conventional "Mexican hat potential," i.e., the SMH. The rest of the sequential Higgs bosons remain approximately pure doublets acquiring small tadpoles via mixing, which is generally suppressed by $1 / M_{x}^{2}$.

Let us presently anticipate the main discussion and illustrate how this setup operates for the top-bottom subsystem. There, we have the Yukawa interactions (see also Ref. [12]):

$$
\mathcal{L}_{\text {yuk }}=-g\left(\bar{t}_{\mathrm{L}}, \bar{b}_{\mathrm{L}}\right) \tilde{H}_{0} t_{\mathrm{R}}-g\left(\bar{t}_{\mathrm{L}}, \bar{b}_{\mathrm{L}}\right) H_{b} b_{\mathrm{R}}
$$

The top mass determines the common Yukawa coupling to be $g=m_{t} / v \simeq 1$.

The $b$ quark then receives its mass from $H_{b}$. By assuming mixing $M_{b}^{2} H_{b}^{\dagger} H_{b}-\mu_{b}^{2} H_{0}^{\dagger} H_{b}+$ H.c., we find the induced tadpole VEV to be $\left\langle H_{b}\right\rangle=\left(0, v_{b}\right)$, where $v_{b}=v \mu_{b}^{2} / M_{b}^{2}$. This implies that the $b$-quark mass is

$$
m_{b}=g v \frac{\mu_{b}^{2}}{M_{b}^{2}}=m_{t} \frac{\mu_{b}^{2}}{M_{b}^{2}}
$$

while the Higgs boson mass is ${ }^{2}$

$$
-M_{0}^{2}=M_{H}^{2}-\frac{\mu_{b}^{4}}{M_{b}^{2}}
$$

To avoid fine-tuned cancellations between the two terms on the rhs of the above equation, we anticipate that

$$
\frac{\mu_{b}^{2}}{M_{b}} \lesssim 100 \mathrm{GeV}
$$

\footnotetext{
${ }^{2}$ Here and throughout the paper, we will use $M_{x}$ to denote Higgs masses and $m_{x}$ to denote fermion masses.
}

providing us with the estimate for a natural value of $M_{b}$ :

$$
M_{b} \lesssim \frac{m_{t}}{m_{b}} \cdot 100 \mathrm{GeV} \simeq 3.5 \mathrm{TeV} .
$$

This is not a firm prediction. ${ }^{3}$ Another possible way of parametrizing the low-scale fine-tuning would be to assume that all $\mu$ parameters are at the electroweak scale, for instance, $\mu=100 \mathrm{GeV}$. We will use these two tuning criteria later as benchmarks for comparison. Furthermore, we can certainly have a lighter $M_{b}$ with less fine-tuning. A mass $M_{b} \sim 380 \mathrm{GeV}$ was previously obtained in a scheme in which the $H_{b}$ with $g=1$ drives a Coleman-Weinberg potential for the $\mathrm{SMH}$, and this has not to our knowledge been ruled out by the LHC [5], though it certainly can be.

This simple scheme is consistent with flavor-physics constraints, as discussed below. Interestingly, flavor dynamics is not then a fundamental consequence of the Yukawa coupling matrices as in the SM, but rather the lowenergy suppression of flavor-changing neutral currents (FCNCs) is a consequence of the heaviness of these states-we also find some natural limits in which the structure of the theory is simplified and in which the phenomenological constraints are easy to understand.

The lightest doublets beyond SMH are $H_{b}$ associated with the $b$ quark, $H_{\tau}$ associated with $\tau$, and $H_{c}$ associated with charm (and possibly $H_{c t}$ and $H_{t c}$, depending on how the Cabibbo-Kobayashi-Maskawa (CKM) matrix is generated, as we will see later). Depending on mixing assumptions, $H_{\tau}$ could, in principle, be lighter than $H_{b}$. We thus strongly encourage LHC experimentalists to consider searching for these objects, and we discuss collider phenomenology in Secs. IVA and IV B.

At the other end of the spectrum, we have the physics of neutrinos and charged leptons. In the scalar democracy, there are three alternatives for the neutrino mass mechanism. For example, where neutrinos are Dirac, their masses are generated like any other fermion in the present model. The Yukawa couplings for the neutrinos are similar to those for quarks and leptons, and the appropriate neutrino mass terms are generated by the mixing of the dormant and SM Higgses. We generate neutrino mixings and small neutrino masses by having ultralarge and positive $M^{2}$ for the neutrino-Higgs fields in the matrix, e.g.,

$$
m_{\nu_{\alpha}} \sim g_{\ell} v \frac{\mu_{\alpha}^{2}}{M_{\nu_{\alpha}}^{2}}
$$

\footnotetext{
${ }^{3} \mathrm{~A}$ more detailed and rigorous treatment of the $(t, b)$ subsystem with $H_{b}$, which can be described by certain simple custodial symmetries, is given in Ref. [12]. If we simply set the input $M_{H}^{2}=0$, we obtain $M_{b} \approx 5.5 \mathrm{TeV}$; however, mixing effects with other lower-mass Higgses such as $H_{\tau}$ will lower this to approximately $3.5 \mathrm{TeV}$. In general, $M_{b} \approx 5.5 \mathrm{TeV}$ remains as a stand-alone $H_{b}$ fine-tuning upper limit, but more Higgs doublets push this mass scale down.
} 
For neutrino masses of order $10^{-2} \mathrm{eV}$, we thus have $M_{\nu_{\alpha}} \sim 10^{11} \mathrm{GeV}$, too heavy for any observable collider or flavor signature. Notwithstanding, the scalar democracy scenario may also lead to Majorana neutrinos via type-I and type-II seesaw mechanisms. In the type-I seesaw case, a light sterile neutrino is predicted, with mass in the kiloelectron-volt to giga-electron-volt range. A general discussion of all these possibilities will be provided in Sec. VA.

\section{Scalar democracy and counting scalars}

If we assume one bound state per fermion pair at some high scale $\Lambda$, then we can count the number of composite scalars in the theory.

All of the SM matter fields can be represented by 48 twocomponent left-handed spinors, $\psi_{A}^{i}$. This includes all the left-handed and anti-right-handed fermions. We can collect these into a large global $\mathrm{SU}(48) \times \mathrm{U}(1)$ multiplet, corresponding to the global symmetry, assuming that the new dynamics are blind to the SM gauge interactions. We emphasize that this is a dynamical symmetry, and familiar grand unified theories (GUTs) that contain only the SM fermions will be gauged subgroups of this SU(48). Here, the indices $(i, j)$ run over all the 48 flavor, doublet, and color degrees of freedom of the SM fermions.

The most general scalar-field bilinear interaction we can construct of these fields is

$$
\epsilon^{A B} \psi_{A}^{i} \psi_{B}^{j} \Theta_{i j}+\text { H.c., }
$$

where $\Theta_{i j}$ transforms as the symmetric $\mathbf{1 1 7 6}$ representation of the $\mathrm{SU}(48) \times \mathrm{U}(1)$ [this is analogous to the sextet representation of $\mathrm{SU}(3)$ ]. The field $\Theta_{i j}$ contains many complex scalar fields with assorted quantum numbers, including baryon and lepton number, color, and weak charges.

To make contact with the SM fields, we consider the usual 24 left-handed quarks and leptons, $\Psi_{\mathrm{L}, i}$, and the 24 right-handed counterparts, $\Psi_{\mathrm{R}, \hat{i}}$. The index $i$ now runs over the chiral $\mathrm{SU}(24)_{\mathrm{L}}$ and $\hat{i}$ over the chiral $\mathrm{SU}(24)_{\mathrm{R}}$ subgroups of SU(48). With this notation, we can construct three interactions with bilinear fermion fields,

$$
\Phi_{\widehat{i j}} \bar{\Psi}_{L}^{i} \Psi_{R}^{\widehat{j}}+\Omega_{i j} \bar{\Psi}_{L}^{i} \Psi_{R}^{j C}+\hat{\Omega}_{i j} \bar{\Psi}_{R}^{\hat{i}} \Psi_{L}^{\hat{j} C}+\text { H.c. }
$$

where $\Phi_{i \hat{j}}$ is the $\left(\mathbf{2 4}_{\mathrm{L}}, \mathbf{2 4}_{\mathrm{R}}\right)$ complex scalar field with $24^{2}=576$ complex degrees of freedom. $\Omega$ and $\hat{\Omega}$ are the symmetric 300 representation of $\mathrm{SU}(24)_{\mathrm{L}}$ and $\mathrm{SU}(24)_{\mathrm{R}}$, respectively. ${ }^{4}$ Thus we have $\Phi(576)+\Omega(300)+\hat{\Omega}(300)=$

\footnotetext{
${ }^{4}$ Notation: if $\Psi_{\mathrm{L}}^{j}=\frac{1-\gamma^{5}}{2} \Psi^{j}$ is a left-handed spinor transforming as a 24 under $\mathrm{SU}(24)_{\mathrm{L}}$, then $\left(\Psi_{\mathrm{L}}^{j}\right)^{C}=i \gamma^{2} \gamma^{0} \frac{\left(1-\gamma^{5}\right)^{*}}{2}\left(\Psi^{j}\right)^{*}=$ $\left(i \gamma^{2} \gamma^{0}\right) \frac{1-\gamma^{5}}{2}\left(-i \gamma^{2} \gamma^{0}\right) \Psi^{j C}=\frac{1+\gamma^{5}}{2} \Psi^{j C}=\left(\Psi^{j C}\right)_{\mathrm{R}} \equiv \Psi_{\mathrm{R}}^{j C}$ (in the notation of Bjorken and Drell) transforms as a $\overline{\mathbf{2 4}}$ under $\mathrm{SU}(24)_{\mathrm{L}}$ and has a $\frac{1+\gamma^{5}}{2}$ projection.
}

$\Theta(1176)$, matching the degrees of freedom of $\Theta_{i j}$. Here, $\Omega_{i j}$ and $\hat{\Omega}_{i j}$ are the analogues of Majorana masses and carry fermion number, while $\Phi$ contains fermion number neutral fields, such as Higgs fields, in addition to $(B-L)$ leptoquark multiplets.

The $\Phi, \Omega$, and $\hat{\Omega}$ fields can be viewed as the "composite fields" arising from a NJL model effective description of the new forces. Consider just the $\mathrm{SU}(24)_{\mathrm{L}} \times \mathrm{SU}(24)_{\mathrm{R}} \times$ $\mathrm{U}(1) \times \mathrm{U}(1)_{A}$ invariant NJL model,

$$
-\frac{g^{2}}{M^{2}}\left(\bar{\Psi}_{\mathrm{L}}^{i} \Psi_{\mathrm{R}}^{j}\right)\left(\bar{\Psi}_{\mathrm{R}, i} \Psi_{\mathrm{L}, j}\right)
$$

where the negative sign denotes an attractive interaction in the potential. It should be noted that we can equally well write current-current (and tensor-tensor) interactions, mediated by heavy spin-1 bosons (or Pauli-Fierz spin-2 gravitons); these will generally contain scalar channels and will Fierz rearrange to effectively reduce to Eq. (13) with the attractive signs. There also exist the possibility of the NJL models

$$
-\frac{g^{2}}{M^{2}}\left(\bar{\Psi}_{\mathrm{L}}^{i} \Psi_{\mathrm{R}}^{C j}\right)\left(\bar{\Psi}_{\mathrm{R}, i}^{C} \Psi_{\mathrm{L}, j}\right) \quad \text { or } \quad(\mathrm{R} \leftrightarrow \mathrm{L}),
$$

which lead to the composite bosons $\Omega$ and $\hat{\Omega}$. Such universal master interactions may arise as subsectors of more general gravitational scattering amplitudes with many other effects near the Planck scale, $M \sim M_{\text {Planck }}$, including gravitational instantons or, at lower-energy scales, e.g., from a strong bigravity force. Equation (13) by itself can therefore be a starting point of a discussion of a dynamically generated extended Higgs boson spectrum.

The first step to solving an NJL theory would be to factorize the interaction of Eq. (13) by introducing auxiliary scalar fields. This leads to the equation we started with, Eq. (12), in which $\Phi, \Omega$, and $\hat{\Omega}$ are auxiliary fields. The universal interaction will bind fermion pairs into scalars that are bound states of ordinary quarks and leptons and will generate a plethora of Higgs doublets. These bound states will have a universal Yukawa coupling $g$ at the scale $M^{2}$. Moreover, with $g$ taking on a near-but-subcritical value, these bound states will generally have large positive masses but can be tuned to be lighter than $M$.

Symmetry-breaking effects will be required to split the spectroscopy, including the SMH down to its observed negative mass term. All other doublets remain heavy but will mix. The problem of solving an NJL model in the large- $N$ fermion loop approximation, or equivalently by the $\mathrm{RG}$, is discussed in detail in Refs. [7,8].

If $g$ is supercritical, then some or all multiplets will acquire negative renormalized masses, $M^{2}(\mu \rightarrow 0)<0$ and the theory develops a vacuum instability. For example, the field $\Phi^{i j}$ with a supercritical coupling will generally condense into a diagonal $\operatorname{VEV},\left\langle\Phi_{i j}\right\rangle=V \delta_{i j}$, and this 
would become a spontaneously broken $\Sigma$ model of $\mathrm{SU}(24)_{\mathrm{L}} \times \mathrm{SU}(24)_{\mathrm{R}} \times \mathrm{U}(1) \times \mathrm{U}(1)_{A}$. In this supercritical case, all the fermions would acquire large, diagonal constituent masses of order $g V$, inconsistent with observation.

The structure we have just outlined, even if subcritical, will contain many composite Higgs doublets. While we want to have a reasonably deep binding of these scalars, the system must be near-to- but subcritical such that no large VEVs will form. We would then expect a positive, diagonal mass-squared matrix among the many composite scalar states, and all fermions would be massless. However, the effect of mixing, i.e., off-diagonal mass terms, can arise from "extended interactions" in analogy to "extended technicolor" or latticized extra dimensions.

Exactly how the scalar mass spectrum is generated is beyond the scope of our present discussion. We will simply assume such a spectrum of masses and mixings between the bound-state scalars that allows for a light sector from the SMH to multi-tera electron volt scales exists and extends up to the highest scales. We assume that $\Omega_{i j}, \hat{\Omega}_{i j}$, and the color-carrying weak doublets have very large positive $M^{2}$, and therefore we will ignore them.

Let us examine the quantum numbers of the spectrum of states in the $\Phi^{i j}$ system. There are $24 \times 24=576$ composite complex scalars, and these devolve into the following states upon gauging the fermions:

(i) $9 \times\left(\mathbf{1}, \mathbf{2}, \frac{1}{2}\right) \sim \bar{Q}_{\mathrm{L}} U_{\mathrm{R}} ; 3^{2} \times 1 \times 2=18$ complex degrees of freedom $(\mathrm{DoF})$,

(ii) $9 \times\left(\mathbf{1}, \mathbf{2},-\frac{1}{2}\right) \sim \bar{Q}_{\mathrm{L}} D_{\mathrm{R}} ; 3^{2} \times 1 \times 2=18$ complex DoF,

(iii) $9 \times\left(\mathbf{1}, \mathbf{2}, \frac{1}{2}\right) \sim \bar{L}_{\mathrm{L}} N_{\mathrm{R}} \quad$ leptonic; $\quad 3^{2} \times 1 \times 2=18$ complex DoF,

(iv) $9 \times\left(\mathbf{1}, \mathbf{2},-\frac{1}{2}\right) \sim \bar{L}_{\mathrm{L}} E_{\mathrm{R}} \quad$ leptonic; $3^{2} \times 1 \times 2=18$ complex DoF,

(v) $9 \times\left(\mathbf{8}, \mathbf{2}, \pm \frac{1}{2}\right) \sim \bar{Q}_{\mathrm{L}} \lambda^{a} U_{\mathrm{R}}\left[D_{\mathrm{R}}\right] ; \quad 3^{2} \times 8 \times 2 \times 2=$ 288 complex DoF,

(vi) $9 \times\left(\mathbf{3}, \mathbf{2}, \frac{1}{6}\left[-\frac{5}{6}\right]\right) \sim \bar{L}_{\mathrm{L}} U_{\mathrm{R}}\left[D_{\mathrm{R}}\right] ; \quad 3^{2} \times 3 \times 2 \times 2=$ 108 complex DoF,

(vii) $9 \times\left(\overline{\mathbf{3}}, 2,-\frac{1}{6}\left[-\frac{7}{6}\right]\right) \sim \bar{Q}_{\mathrm{L}} N_{\mathrm{R}}\left[E_{\mathrm{R}}\right] ; \quad 3^{2} \times 3 \times 2 \times 2=$ 108 complex DoF,

where the brackets denote the SM quantum numbers. The first four entries in the above list are identified with the 36 Higgs doublets, 18 for the quark and 18 for the lepton sector.

\section{Renormalization group fixed points}

If we consider the 18 scalars in the quark sector, ignoring their masses and electroweak (EW) charges, we will have a Yukawa interaction at the scale $\Lambda$ that is $\mathrm{SU}(6)_{\mathrm{L}} \times \mathrm{SU}(6)_{\mathrm{R}}$ invariant and of the form

$$
g \bar{\Psi}_{\mathrm{L}} \Sigma \Psi_{\mathrm{R}}+\text { H.c. },
$$

where $\Psi=(u, d, c, s, t, b)$ and $\Sigma$ is a $6 \times 6$ complex matrix composed of 18 doublets. The renormalization group equation for $g$ is then determined to be

$$
\left(16 \pi^{2}\right) \frac{\mathrm{d} g}{\mathrm{~d} \ln (\mu)}=g\left(9 g^{2}-8 g_{3}^{2}-\kappa\right)
$$

at one-loop order, where $\kappa$ includes the smaller electroweak corrections [which breaks the $\mathrm{SU}(6)_{\mathrm{L}} \times \mathrm{SU}(6)_{\mathrm{R}}$ invariance]. This describes the running of $g$ down to scales at which the various Higgs doublets decouple. Likewise, we have an equation for the lepton sector, $g \rightarrow g_{\ell}$, where we drop the quark's $-8 g_{3}^{2}$ term.

Assuming the Planck mass corresponds to a Landau pole in $g$ and that all Higgs bosons are active down to the electroweak scale, then we derive $g \simeq 0.93$ and $g_{\ell} \simeq 0.71$ (this also leads to some "fine structure" as the electroweak terms in $\kappa$ split the degeneracy between the Yukawa couplings for the up- and down-type quarks; the full details of this are beyond the scope of this paper).

This result implies a top-quark mass of approximately $161 \mathrm{GeV}$. This is the prediction of the modified RG fixed point (equivalent to a focus point) of Ref. [21] including additional Higgs bosons and represents a significant improvement over the original minimal top condensation models [7]. This prediction is robust with respect to the precise values, $\Lambda$ and $g(\Lambda)$. If we then include the masses of the heavier Higgs bosons (as discussed below) and decouple them at their thresholds, the prediction will increase, and we expect it can converge on the observed topquark mass.

Grand unification is greatly complicated in scalar democracy, but it is implementable. We relegate such an investigation to future work.

\section{LOW-ENERGY EFFECTIVE THEORY}

The observed Higgs boson must reside among the color singlet $\bar{Q}_{\mathrm{L}} q_{\mathrm{R}}$ doublets. We proceed under the simplifying assumption that the SM Higgs doublet, $H_{0}$, can be identified with the doublet that couples to the fermionic combination of a top-quark pair,

$$
\left(\bar{t}_{\mathrm{L}}, \bar{b}_{\mathrm{L}}\right) t_{\mathrm{R}} \sim H_{0}
$$

This is the unique logical choice, as it has the largest Yukawa coupling in the standard model and our theory dictates that all quarks will have this universal coupling. This, in part, recovers the top-condensation models [6-8].

We further reduce the scope of the problem by assuming all of the dormant Higgs doublets apart from the 36 color singlet $\bar{Q}_{\mathrm{L}} q_{\mathrm{R}}$ and $\bar{L}_{\mathrm{L}} \ell_{\mathrm{R}}$ doublets are arbitrarily heavy and therefore decoupled from the low-energy effective theory. Although the phenomenology of these other scalars could be very interesting, this framework does not provide any insight on their mass scale. Besides, we will assume for concreteness that the neutrino mass mechanism is the same as for charged fermions and comment on alternatives in Sec. VA. 
At this point, it is convenient to resort to a common notation for the individual Higgs bosons. Below the scale $\Lambda$, the effective theory we are considering is a modification of the SM where the Higgs sector has been replaced by

$$
\begin{aligned}
\mathcal{L} \supset & \left|D_{\mu} H^{\prime}\right|^{2}-V\left(H^{\prime}\right)-g \bar{Q}_{\mathrm{L}}^{\prime i} H_{i j}^{\prime d} D_{\mathrm{R}}^{\prime j}-g \bar{Q}_{\mathrm{L}}^{\prime i} \tilde{H}_{i j}^{\prime u} U_{\mathrm{R}}^{\prime j} \\
& -g_{\ell} \bar{L}_{\mathrm{L}}^{\prime i} H_{i j}^{\prime e} E_{\mathrm{R}}^{\prime j}-g_{\ell} \bar{L}_{\mathrm{L}}^{\prime i} \tilde{H}_{i j}^{\prime \prime} N_{\mathrm{R}}^{\prime j}+\text { H.c. }
\end{aligned}
$$

The generational indices of the fermions are labeled by indices $i, j$, and the primes indicate that we are working in the gauge eigenbasis. The Higgs doublets are denoted by $H_{i j}^{\prime f}$ in their flavor eigenbasis, where $f=u, d, \nu, e$ represents the fermion type, which acquires mass from the doublet and $i, j$ the generations they couple to. Each doublet has an upper charged component and a lower neutral component, $H_{x}=\left(h_{x}^{+}, h_{x}^{0}\right)$, and we employ the charge conjugation convention $\tilde{H}=i \sigma_{2} H^{*}$. All fermionic masses and mixings are due to the VEVs of the Higgs bosons. The RG improved universality implies that all quarks have a Yukawa coupling $g \simeq 1$ and leptons $g_{\ell} \simeq 0.7$.

\section{A. Higgs potential}

Formally, we can define the 36 Higgs doublets as a "vector," where we separate out the (mostly) SM-like Higgs: $\left(H_{0}^{\prime}, H_{a}^{\prime}\right)$. Thus, in addition to the SM-like Higgs, $H_{0}^{\prime}$, we have 35 doublets represented as $H_{a}^{\prime}=\left(H_{1}^{\prime}, \ldots, H_{35}^{\prime}\right)$. Here, we define a new notation and apologize for that. Nevertheless, this index notation will only be applied in this subsection. In the remainder of the paper, we revert to the notation $H_{i j}^{f}$.

We may write out the multi-Higgs boson potential as

$$
\begin{aligned}
V= & M_{H}^{2} H_{0}^{\prime \dagger} H_{0}^{\prime}+\frac{\lambda}{2}\left|H_{0}^{\prime}\right|^{4}+H_{a}^{\prime \dagger} M_{a b}^{2} H_{b}^{\prime} \\
& -\left(H_{a}^{\prime \dagger} \mu_{a}^{2} H_{0}^{\prime}+\text { H.c. }\right),
\end{aligned}
$$

where the positive quartic interactions of the dormant Higgses are taken to be negligible due to their large masses. ${ }^{5}$ Thus, the Higgs mixing is determined by the mass matrix. The mass terms that mix $H_{a}^{\prime}$ with $H_{0}^{\prime}$ can be viewed as a vector, $\mu_{a}^{2}=\left(\mu_{1}^{2}, \ldots, \mu_{35}^{2}\right)$, and $M_{a b}^{2}$ is a $35 \times 35$ Hermitian mass matrix among the heavy scalars, which is taken to have positive eigenvalues.

It is important to note that there are two distinct types of mixing in this model sourced by $\mu_{a}^{2}$ and the off-diagonal part of $M_{a b}^{2}$, respectively. The former is responsible for mixing the SMH into all the Higgs flavor states, while the latter introduces mixing between the dormant Higgses. We will regard the off-diagonal elements, $\mu_{a}^{2}$, as perturbatively small when compared to $M_{a b}^{2}$.

\footnotetext{
${ }^{5} \mathrm{~A}$ universal quartic coupling is another option for the quartic terms that confine Higgs mixing to the mass matrix.
}

The negligible quartic couplings leads to a mass degeneracy of the charged and neutral Higgs components of the dormant doublets. Therefore, retaining the symmetrybreaking mass terms, the dormant Higgs fields will acquire tadpole VEVs via the $\mu_{a}^{2} H_{a}^{\prime \dagger} H_{0}^{\prime}$ mixings, but their fluctuating fields will be degenerate doublets.

The Higgs mass terms are diagonalized at leading order (LO) in $M_{H}^{2}, \mu_{a}^{2} / M_{a b}^{2}$ by going to the basis defined by

$$
\begin{aligned}
& H_{a}^{\prime}=H_{a}+M_{a b}^{-2} \mu_{b}^{2} H_{0}+\cdots, \\
& H_{0}^{\prime}=H_{0}-\mu_{a}^{2 *} M_{a b}^{-2} H_{b}+\cdots,
\end{aligned}
$$

where $H_{0}, H_{a}$ defines the physical Higgs doublets before electroweak symmetry breaking. After rotating away the $\mu_{a}$ mixing, the equations of motion for the VEVs of the Higgs fields read

$$
\begin{aligned}
-M_{0}^{2}\left\langle H_{0}\right\rangle+\lambda\left|\left\langle H_{0}\right\rangle\right|^{2}\left\langle H_{0}\right\rangle & =0, \\
M_{a b}^{2}\left\langle H_{b}\right\rangle & =0,
\end{aligned}
$$

where

$$
-M_{0}^{2}=\left(M_{H}^{2}-\mu_{a}^{2} M_{a b}^{-2} \mu_{b}^{2}\right) \simeq-(88.4 \mathrm{GeV})^{2}
$$

is the mass term for the SMH. The Higgs tadpoles are thus given by

$$
\left\langle H_{0}\right\rangle=\left(\begin{array}{l}
0 \\
v
\end{array}\right) \text { and }\left\langle H_{a}\right\rangle=\mathbf{0},
$$

where $v^{2}=-M_{0}^{2} / \lambda \simeq(174 \mathrm{GeV})^{2}$ is the SM VEV. Only the physical SMH doublet gets a VEV, and all fermions get their masses through couplings to it, thereby recovering SM couplings. From our assumptions of the Higgs potential, we ignore further mixing between the massive Higgs bosons. The dormant Higgses constitute degenerate doublets, and $H_{0}$ is directly identified with the $\mathrm{SMH}$, its components being the physical Higgs and the three EW Goldstone bosons. Eq. (20) shows how $H_{0}$ is mixed into all the Higgs flavor states. Accordingly, the dormant Higgses acquire small VEVs which in turn produces the fermion masses in our model.

\section{B. Fermion masses and mixing}

The fermions acquire their masses from the Yukawa couplings as shown in Eq. (18). In general, the mass matrices for the fermions are determined by the VEVs of the corresponding Higgs flavor eigenstates,

$$
m_{i j}^{f} \equiv\left[\mathcal{L}_{f}^{\dagger} \operatorname{diag}\left(m_{1}^{f}, m_{2}^{f}, m_{3}^{f}\right) \mathcal{R}_{f}\right]_{i j}=g_{f}\left\langle h_{i j}^{\prime f, 0}\right\rangle,
$$

where $g_{f}$ is the Higgs coupling to quarks $(g)$ or leptons $\left(g_{\ell}\right)$; $\mathcal{L}_{f}$ and $\mathcal{R}_{f}$ are unitary matrices which rotate left- and righthanded fermions, respectively, from the gauge to the mass 
basis. We will proceed to determine the physical couplings between the fermions and the new Higgses, assuming the Higgs potential outlined in the previous section, and furthermore take the limit where the mass matrix, $M_{a b}^{2}$, of the dormant Higgses is diagonal, thereby eliminating dormant-Higgs mixing.

First, we focus on the quarks, which have mass eigenstates given by

$$
\begin{aligned}
& U_{\mathrm{L}}=\left(\begin{array}{c}
u_{\mathrm{L}} \\
c_{\mathrm{L}} \\
t_{\mathrm{L}}
\end{array}\right)=\mathcal{L}_{u} Q_{\mathrm{L}}^{\prime \prime}, \quad U_{\mathrm{R}}=\left(\begin{array}{c}
u_{\mathrm{R}} \\
c_{\mathrm{R}} \\
t_{\mathrm{R}}
\end{array}\right)=\mathcal{R}_{u} U_{\mathrm{R}}^{\prime}, \\
& D_{\mathrm{L}}=\left(\begin{array}{c}
d_{\mathrm{L}} \\
s_{\mathrm{L}} \\
b_{\mathrm{L}}
\end{array}\right)=\mathcal{L}_{d} Q_{\mathrm{L}}^{\prime d}, \quad D_{\mathrm{R}}=\left(\begin{array}{c}
d_{\mathrm{R}} \\
s_{\mathrm{R}} \\
b_{\mathrm{R}}
\end{array}\right)=\mathcal{R}_{d} D_{\mathrm{R}}^{\prime} .
\end{aligned}
$$

The couplings between the down-type quarks and the neutral components of the dormant Higgses, in the mass eigenbasis, are given by ${ }^{6}$

$$
\begin{aligned}
\mathcal{L} \supset & -\bar{D}_{\mathrm{L}}\left[g \mathcal{L}_{d}\left(\begin{array}{ccc}
h_{d}^{0} & h_{d s}^{0} & h_{d b}^{0} \\
h_{s d}^{0} & h_{s}^{0} & h_{s b}^{0} \\
h_{b d}^{0} & h_{b s}^{0} & h_{b}^{0}
\end{array}\right) \mathcal{R}_{d}^{\dagger}\right. \\
& \left.+\left(\begin{array}{ccc}
m_{d} & & \\
& m_{s} & \\
& & m_{b}
\end{array}\right)\left(1+\frac{h}{v}\right)\right] D_{\mathrm{R}},
\end{aligned}
$$

and likewise for the up-type quarks

$$
\begin{aligned}
\mathcal{L} \supset & -\bar{U}_{\mathrm{L}}\left[g \mathcal{L}_{u}\left(\begin{array}{ccc}
h_{u}^{0} & h_{u c}^{0} & h_{u t}^{0} \\
h_{c u}^{0} & h_{c}^{0} & h_{c t}^{0} \\
h_{t u}^{0} & h_{t c}^{0} & {[h]}
\end{array}\right) \mathcal{R}_{u}^{\dagger}\right. \\
& \left.+\left(\begin{array}{ccc}
m_{u} & & \\
& m_{c} & \\
& & m_{t}
\end{array}\right)\left(1+\frac{h}{v}\right)\right] U_{\mathrm{R}},
\end{aligned}
$$

where $[h]=-\mu_{a}^{2 *} M_{a b}^{-2} h_{b}^{0}$ arises from the feedback on the $\mathrm{SMH}$. Similarly, in the lepton sector, we set the mass eigenstates to be

\footnotetext{
${ }^{6}$ For convenience, we have identified, e.g., $H_{23}^{d}=H_{s b}$. We apologize for the notational inefficiencies.
}

$$
\begin{gathered}
N_{\mathrm{L}}=\left(\begin{array}{c}
\nu_{1 \mathrm{~L}} \\
\nu_{2 \mathrm{~L}} \\
\nu_{3 \mathrm{~L}}
\end{array}\right)=\mathcal{L}_{\nu} L_{\mathrm{L}}^{\prime \nu}, \quad N_{\mathrm{R}}=\left(\begin{array}{c}
\nu_{1 \mathrm{R}} \\
\nu_{2 \mathrm{R}} \\
\nu_{3 \mathrm{R}}
\end{array}\right)=\mathcal{R}_{\nu} N_{\mathrm{R}}^{\prime}, \\
E_{\mathrm{L}}=\left(\begin{array}{c}
e_{\mathrm{L}} \\
\mu_{\mathrm{L}} \\
\tau_{\mathrm{L}}
\end{array}\right)=\mathcal{L}_{e} L_{\mathrm{L}}^{\prime e}, \quad E_{\mathrm{R}}=\left(\begin{array}{c}
e_{\mathrm{R}} \\
\mu_{\mathrm{R}} \\
\tau_{\mathrm{R}}
\end{array}\right)=\mathcal{R}_{e} E_{\mathrm{R}}^{\prime},
\end{gathered}
$$

which gives interactions with the neutral Higgs bosons of the following form:

$$
\begin{aligned}
\mathcal{L} \supset & -\bar{E}_{\mathrm{L}}\left[g_{\ell} \mathcal{L}_{e}\left(\begin{array}{ccc}
h_{e}^{0} & h_{e \mu}^{0} & h_{e \tau}^{0} \\
h_{\mu e}^{0} & h_{\mu}^{0} & h_{\mu \tau}^{0} \\
h_{\tau e}^{0} & h_{\tau \mu}^{0} & h_{\tau}^{0}
\end{array}\right) \mathcal{R}_{e}^{\dagger}\right. \\
& \left.+\left(\begin{array}{ccc}
m_{e} & \\
& m_{\mu} & \\
& & m_{\tau}
\end{array}\right)\left(1+\frac{h}{v}\right)\right] E_{\mathrm{R}}
\end{aligned}
$$

and

$$
\begin{aligned}
\mathcal{L} \supset & -\bar{N}_{\mathrm{L}}\left[g_{\ell} \mathcal{L}_{\nu}\left(\begin{array}{ccc}
h_{1}^{0} & h_{12}^{0} & h_{13}^{0} \\
h_{21}^{0} & h_{2}^{0} & h_{23}^{0} \\
h_{31}^{0} & h_{32}^{0} & h_{3}^{0}
\end{array}\right) \mathcal{R}_{\nu}^{\dagger}\right. \\
& \left.+\left(\begin{array}{lll}
m_{1} & & \\
& m_{2} & \\
& & m_{3}
\end{array}\right)\left(1+\frac{h}{v}\right)\right] N_{\mathrm{R}} .
\end{aligned}
$$

A similar construction for the coupling of the fermions to the charged Higgses follows straightforwardly. We observe that $h$, the neutral component of $H_{0}$, is completely indistinguishable from the SMH.

From the previously outlined assumptions on the Higgs sector, we are able to estimate the masses of the dormant Higgses. Using Eqs. (24) and (20), along with diagonal dormant mass matrix, we find that the fermion mass matrix is related to the Higgs mass terms by

$$
m_{i j}^{f}=\left(\frac{g_{f}}{g}\right) \frac{\mu_{i j}^{f 2}}{M_{i j}^{f 2}} m_{t} .
$$

To avoid a large fine-tuning in the SMH mass term, we expect $\mu^{4} / M^{2} \lesssim(100 \mathrm{GeV})^{2}$. We thus arrive at the estimate

$$
M_{i j}^{f} \lesssim\left(\frac{g_{f}}{g}\right) \frac{m_{t}}{m_{i j}^{f}} \cdot 100 \mathrm{GeV}
$$


We therefore expect the dormant Higgs masses to be inversely proportional to the corresponding entry in the fermion mass matrix. Once we have made an ansatz for the fermion rotation matrices, $\mathcal{L}_{f}, \mathcal{R}_{f}$, this provides us with an estimate for the Higgs masses. The bound shown in Eq. (32) should merely be viewed as a guideline, as it is based on a fine-tuning argument.

Before we proceed to review two important limiting cases in the next section, we first define our notation: the CKM matrix is given by $V_{\mathrm{CKM}}=\mathcal{L}_{u} \mathcal{L}_{d}^{\dagger}$, and likewise the Pontecorvo-Maki-Nakagawa-Sakata (PMNS) matrix is given by $V_{\mathrm{PMNS}}=\mathcal{L}_{\nu} \mathcal{L}_{e}^{\dagger}$. In the limit of negligible dormant Higgs mixing, the required input to determine the Yukawa couplings is the set of mass rotations, $\mathcal{L}_{f}$ and $\mathcal{R}_{f}$. These rotation matrices are implicitly generated by the unknown $\mu_{a}^{2}$, which gives rise to the VEV structure we have encoded into the above expressions. Examining Eqs. (26) and (30), we find that the individual matrices $\mathcal{L}_{f}, \mathcal{R}_{f}$ are unobservable in the standard model. However, their combinations form the mixing matrices, $V_{\mathrm{CKM}}$ and $V_{\mathrm{PMNS}}$, which enter in the charged currents of the quark and lepton sectors, respectively. Given sufficient dormant Higgs data, the structure of $\mathcal{L}$ and $\mathcal{R}$ could be determined but at present cannot be determined directly. We must therefore make an ansatz for their form.

\section{C. $\mathcal{R}_{f}=\mathbb{1}_{3}$ and no-mixing limits}

We proceed by considering the various symmetries that arise in the special limit $\mathcal{R}_{f} \rightarrow \mathbb{1}_{3}$ and $m_{q}=0$. These are useful for relaxing rather stringent flavor mixing constraints.

Consider Eq. (26) with $\mathcal{R}_{d}=\mathbb{1}_{3}$ and the strange quark mass set to zero, $m_{s}=0$. Furthermore, decompose the mass matrix of the dormant Higgs fields, $H_{a}$, as a sum of a diagonal $M_{\text {diag }}^{2}$ and an off-diagonal $\delta M^{2}$ Hermitian matrices:

$$
M^{2}=M_{\mathrm{diag}}^{2}+\delta M^{2}
$$

In the case $\delta M^{2}=0$, we find there exists a discrete symmetry, ${ }^{7}$ e.g., reflection of the right-handed strange quark, $s_{R} \rightarrow-s_{R}$ and the corresponding Higgs fields $\left(H_{b s}, H_{s}, H_{d s}\right) \rightarrow-\left(H_{b s}, H_{s}, H_{d s}\right)$. This symmetry is a generalization of the Glashow-Weinberg symmetry [23], and in our present case of many Higgs bosons, this is restrictive.

First, we observe that in general $H_{s}$ will mediate an interaction of the form,

\footnotetext{
${ }^{7}$ This symmetry can only be approximately realized in a realistic part of the parameter space; it is broken by the quark mass terms and the Yukawa coupling between the top quark and the dormant Higgses.
}

$$
\frac{1}{M_{s}^{2}}\left(\bar{s}_{\mathrm{L}}^{\prime} s_{\mathrm{R}}^{\prime}\right)\left(\bar{s}_{\mathrm{R}}^{\prime} s_{\mathrm{L}}^{\prime}\right)
$$

in the gauge basis. In the case that $\mathcal{R}_{d}$ were not unity, this would contain mixed combinations such as

$$
\begin{aligned}
& \frac{1}{M_{s}^{2}}\left|\left(\mathcal{L}_{d, 22} \bar{s}_{\mathrm{L}}+\mathcal{L}_{d, 12} \bar{d}_{\mathrm{L}}\right)\left(\mathcal{R}_{d, 22}^{*} s_{\mathrm{R}}+\mathcal{R}_{d, 12}^{*} d_{\mathrm{R}}\right)\right|^{2}, \\
& \supset \frac{1}{M_{s}^{2}} \mathcal{L}_{d, 22} \mathcal{L}_{d, 12} \mathcal{R}_{d, 22}^{*} \mathcal{R}_{d, 12}^{*}\left(\bar{s}_{\mathrm{L}} d_{\mathrm{R}}\right)\left(\bar{s}_{\mathrm{R}} d_{\mathrm{L}}\right)+\cdots
\end{aligned}
$$

in the flavor basis, which results in $\Delta S=2$ transitions. The $K_{L}-K_{S}$ mass splitting places severe limits on $M_{s} \gtrsim$ $10^{3} \mathrm{TeV}$ for left- and right-handed mixings of the order of the CKM matrix, while the mass estimate of Eq. (32) suggests $M_{s} \lesssim 100 \mathrm{TeV}$. With the discrete symmetry, $\mathcal{R}_{d}=\mathbb{1}_{3}$, no such interactions are generated at tree level, and the tension is substantially alleviated. Moreover, the discrete symmetry forbids the similarly dangerous Higgs mixing term $\delta M_{d s, s d}^{2} H_{d s}^{\dagger} H_{s d}$, which mediates interactions such as $\frac{1}{M^{2}}\left(\bar{d}_{\mathrm{L}} s_{\mathrm{R}}\right)\left(\bar{d}_{\mathrm{R}} s_{\mathrm{L}}\right)$ directly.

At the one-loop level, the Higgs-Higgs box diagrams, with exchange of the full doublet $H_{s}$, produces the effective interaction

$$
\frac{1}{32 \pi^{2}} \frac{1}{M_{s}^{2}}\left(\bar{s}_{\mathrm{L}}^{\prime} \gamma_{\mu} s_{\mathrm{L}}^{\prime}\right)\left(\bar{s}_{\mathrm{L}}^{\prime} \gamma_{\mu} s_{\mathrm{L}}^{\prime}\right)
$$

This operator is not restricted by $\mathcal{R}_{d}=\mathbb{1}_{3}$ but yields a $\Delta S=2$ operator for nontrivial left-handed rotations, $\mathcal{L}_{q}$,

$$
\frac{1}{32 \pi^{2}} \frac{1}{M_{s}^{2}}\left(\mathcal{L}_{d, 22}\right)^{2}\left(\mathcal{L}_{d, 12}^{*}\right)^{2}\left(\bar{s}_{\mathrm{L}} \gamma_{\mu} d_{\mathrm{L}}\right)\left(\bar{s}_{\mathrm{L}} \gamma_{\mu} d_{\mathrm{L}}\right)
$$

Comparing this effective operator with kaon-mixing bounds on the left-handed current [24], we arrive at the limit $M_{s} \gtrsim 60 \mathrm{TeV}$, which is compatible with the mass estimate.

Our model can thus generate CKM mixing, with multiple flavorful Higgs doublets and yet present no large FCNC [no tree-level $\mathcal{O}\left(1 / M_{x}^{2}\right)$ operators] if we make $\mathcal{R}=\mathbb{1}_{3}$. Typical cases include small breaking effects, giving $\mathcal{R} \neq \mathbb{1}_{3}$, and flavor physics remains an important probe in a system like this.

In getting a sense of the model, it is useful to consider the limit in which $\mathcal{L}_{f}, \mathcal{R}_{f}$ are set to unity with $\delta M_{a b}^{2}=0$, which turns off the mixing among dormant Higgses at leading order. We still allow nonzero $\mu_{a}^{2}$ terms that mix the dormant Higgses to the SMH.

Let us focus on the up-quark system cf., Eq. (27). The top quark has acquired its mass by direct coupling to the $\mathrm{SMH}$, which defines the universal quark Yukawa coupling $g=1$. In this limit, we can identify the fields that develop tadpole VEVs and give the light-quark masses, Eq. (20), 
TABLE I. The nonmixing estimates for heavy dormant Higgs bosons masses assuming (1) the level-repulsion feedback on the Higgs mass term is limited to $(100 \mathrm{GeV})^{2}$ for each of the quarks and leptons, hence $M_{q}=(100 \mathrm{GeV})\left(m_{t} / m_{q}\right)$ and $M_{\ell}=(100 \mathrm{GeV})\left(g_{\ell} m_{t} / m_{\ell}\right)$, and (2) $\mu=100 \mathrm{GeV}$ for all mixings, hence $M_{q}=\mu\left(m_{t} / m_{q}\right)^{1 / 2}$ and $M_{\ell}=\mu\left(m_{t} g_{\ell} / g m_{q}\right)^{1 / 2}$. Here, $g=1, g_{\ell}=0.7$, and $v=175 \mathrm{GeV}$. The scalar spectrum relevant to the neutrino mass generation is discussed in more detail in Sec. VA.

\begin{tabular}{|c|c|c|c|}
\hline Higgs field & Fermion mass & Case (1) [TeV] & Case (2) [TeV] \\
\hline$H_{0}^{\prime}=v+\frac{h}{\sqrt{2}}$ & $m_{t}=g v=175 \mathrm{GeV}$ & $m_{H}=0.125$ & $m_{H}=0.125$ \\
\hline$H_{b}^{\prime}=v \frac{\mu^{2}}{M_{b}^{2}}+H_{b}$ & $m_{b}=g v \frac{\mu^{2}}{M_{b}^{2}}=4.5 \mathrm{GeV}$ & $M_{b}=3.9$ & $M_{b}=0.620$ \\
\hline$H_{\tau}^{\prime}=v \frac{\mu^{b}}{M_{\tau}^{2}}+H_{\tau}$ & $m_{\tau}=g_{\ell} v \frac{\mu^{2}}{M_{\tau}^{2}}=1.8 \mathrm{GeV}$ & $M_{\tau}=6.8$ & $M_{\tau}=0.825$ \\
\hline$H_{c}^{\prime}=v \frac{\mu^{2}}{M_{b}^{2}}+H_{c}$ & $m_{c}=g v \frac{\mu^{2}}{M_{c}^{2}}=1.3 \mathrm{GeV}$ & $M_{c}=13.5$ & $M_{c}=1.2$ \\
\hline$H_{\mu}^{\prime}=v \frac{\mu^{2}}{M_{\mu}^{2}}+H_{\mu}$ & $m_{\mu}=g_{\ell} v \frac{\mu^{2}}{M_{\mu}^{2}}=106 \mathrm{MeV}$ & $M_{\mu}=1.2 \times 10^{2}$ & $M_{\mu}=3.4$ \\
\hline$H_{s}^{\prime}=v \frac{\mu^{2}}{M_{s}^{2}}+H_{s}$ & $m_{s}=g v \frac{\mu^{2}}{M_{s}^{2}}=95 \mathrm{MeV}$ & $M_{s}=1.8 \times 10^{2}$ & $M_{s}=4.3$ \\
\hline$H_{d}^{\prime}=v \frac{\mu^{2}}{M_{d}^{2}}+H_{d}$ & $m_{d}=g v \frac{\mu^{2}}{M_{d}^{2}}=4.8 \mathrm{MeV}$ & $M_{d}=3.6 \times 10^{3}$ & $M_{d}=19$ \\
\hline$H_{u}^{\prime}=v \frac{\mu^{2}}{M_{u}^{2}}+H_{u}$ & $m_{u}=g v \frac{\mu^{\frac{d}{2}}}{M_{u}^{2}}=2.3 \mathrm{MeV}$ & $M_{u}=7.6 \times 10^{3}$ & $M_{u}=27$ \\
\hline$H_{e}^{\prime}=v \frac{\mu^{2}}{M_{e}^{2}}+H_{e}$ & $m_{e}=g_{\ell} v \frac{\mu^{2}}{M_{e}^{2}}=0.5 \mathrm{MeV}$ & $M_{e}=2.45 \times 10^{4}$ & $M_{e}=49$ \\
\hline
\end{tabular}

$$
\begin{array}{ll}
H_{c}^{\prime}=H_{c}+\frac{\mu_{c}^{2}}{M_{c}^{2}} H_{0}, & m_{c}=\frac{\mu_{c}^{2}}{M_{c}^{2}} v, \\
H_{u}^{\prime}=H_{u}+\frac{\mu_{u}^{2}}{M_{u}^{2}} H_{0}, & m_{u}=\frac{\mu_{u}^{2}}{M_{u}^{2}} v,
\end{array}
$$

and likewise for the $d, e$, and $\nu$ sectors. In this limit, we can compute the diagonal dormant Higgs masses. All other fields $H_{x}$ have no VEVs in this limit. The full set of dormant Higgs masses are tabulated in Table I under two different case assumptions:

(1) The feedback on the Higgs mass is maximal but limited to $(100 \mathrm{GeV})^{2}$ for each fermion (thus choosing a larger value of $\mu$ for lighter quarks); hence, $M_{q}=\left(m_{t} / m_{q}\right) \cdot 100 \mathrm{GeV}$, and $M_{\ell}=$ $\left(g_{\ell} m_{t} / m_{\ell}\right) \cdot 100 \mathrm{GeV}$.

(2) $\mu=100 \mathrm{GeV}$ for each $\mathrm{SMH}$ mixing term, $M_{q}=$ $\left(m_{t} / m_{q}\right)^{1 / 2} \cdot 100 \mathrm{GeV}$ and $M_{\ell}=\left(g_{\ell} m_{t} / m_{\ell}\right)^{1 / 2}$. $100 \mathrm{GeV}$, which makes only the lightest $H_{b}, H_{\tau}$, and $H_{c}$ have any significant feedback on the SMH mass.

Hence, in the zero mixing angle limit, it is easy to see how the flavor problem is mapped into an inverted mass spectrum of Higgs doublets.

\section{QUARK-SECTOR PHENOMENOLOGY}

Throughout Secs. IV A and IV B, we calculate the LO production cross section of the new physics (NP) processes using Universal FeynRules Output (UFO) format of FEYnRules [25] implemented into MADGRAPH5_AMC@ NLO [26] and applying the NNPDF2.3 parton distribution function set [27]. We implement the five-flavor scheme to implement the perturbative (intrinsic) $b$-quark structure function of the proton. Flavor constraints on the dormant Higgs masses from meson mixing and dedicated searches for flavor change in the charged lepton sector are outlined in Secs. IV C and V B, respectively.

\section{A. Current limits from LHC searches}

The most accessible state at the LHC is the lightest (non$\mathrm{SM}$ ) doublet, which couples to the $b$ quarks, namely, $H_{b}=\left(h_{b}^{+}, h_{b}^{0}\right)$. This behaves as a degenerate isodoublet in which $h_{b}^{+}$and $h_{b}^{0}$ are complex fields. We focus on the neutral component, $h_{b}^{0}$, which has the dominant production process.

For single $h_{b}^{0}$ production, the dominant contribution is via $b$-quark fusion as shown in Fig. 1. Initial-state QCD are resummed to yield the $b$-quark structure function; hence, we study the dominant process $p p(b \bar{b}) \rightarrow h_{b}^{0} \rightarrow b \bar{b}$. Single $h_{b}^{0}$ production via open gluon fusion is perturbatively smaller, and gluon fusion to a bottom-quark loop is negligible. ${ }^{8}$ For us, the coupling of the $b$ quarks to $h_{b}^{0}$ is $g=1$. This implies that $h_{b}^{0}$ will decay approximately to two $b$ quarks with a branching ratio of 1 . A comparison of searches for new resonances decaying into jets containing $b$ hadrons can potentially give exclusion limits for the relevant (multi-tera electron volt) mass regime of $h_{b}^{0}$.

We consider an ATLAS analysis of a dataset collected at $\sqrt{s}=13 \mathrm{TeV}$ and searches for two $b$-tagged jets with an invariant mass $\left(m_{b b}\right)$ in the $0.57-5 \mathrm{TeV}$ range [28]. In the "low" $\left[0.6 \leq m_{b b}(\mathrm{TeV})<1.25\right]$ and "high" $\left[1.25 \leq m_{b b}(\mathrm{TeV}) \leq 5.0\right]$ invariant mass regimes, $24.3 \mathrm{fb}^{-1}$ and $36.1 \mathrm{fb}^{-1}$ of integrated luminosity were analyzed, respectively. The primary aim of this analysis is to search for $Z^{\prime}$ decays to $b$ quarks, and although designed

\footnotetext{
${ }^{8}$ The top loop will also contribute to this process because of the mixing of the SMH with $h_{b}^{0}$. The modified coupling of the top quarks to $h_{b}^{0}$ is approximately $g m_{b} / m_{t}$, cf., Eq. (27).
} 


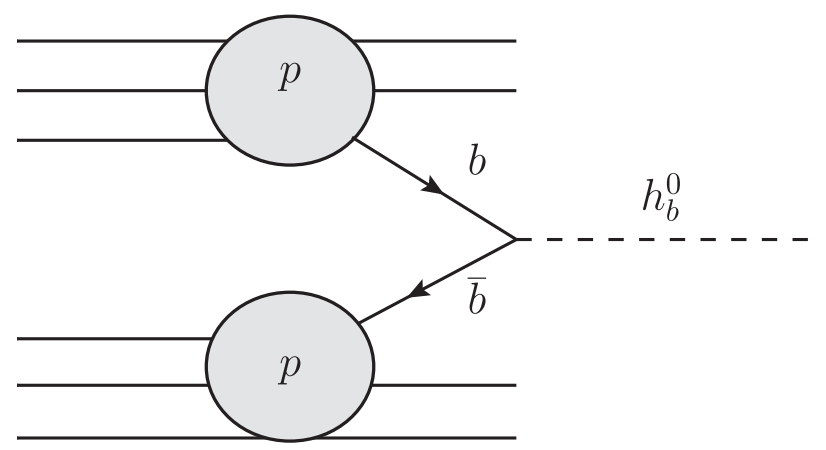

FIG. 1. The dominant contribution to single $h_{b}^{0}$ production is $b$ quark fusion. $h_{b}^{0}$ decays with a branching ratio of approximately 1 to a two- $b$ quark final state.

to place limits on a vector boson rather than a scalar, the final states are the same in both cases, so we expect the acceptance between our model and that of Ref. [28] to be comparable.

The one- and two-sigma regions for the expected upper limit on the cross section of di $-b$ jet production is shown in Fig. 2, as indicated by green and yellow, respectively, while the observed upper limit, at 95\% C.L., is shown by the black circles. There is no significant deviation between observed and expected limits. The details of the analysis used to calculate these curves can be found in the aforementioned reference.

We have not performed a full analysis, including detector effects, to calculate signal cross section, and therefore the uncorrected cross section will likely be a slight overestimate. To mitigate this issue, the LO production cross

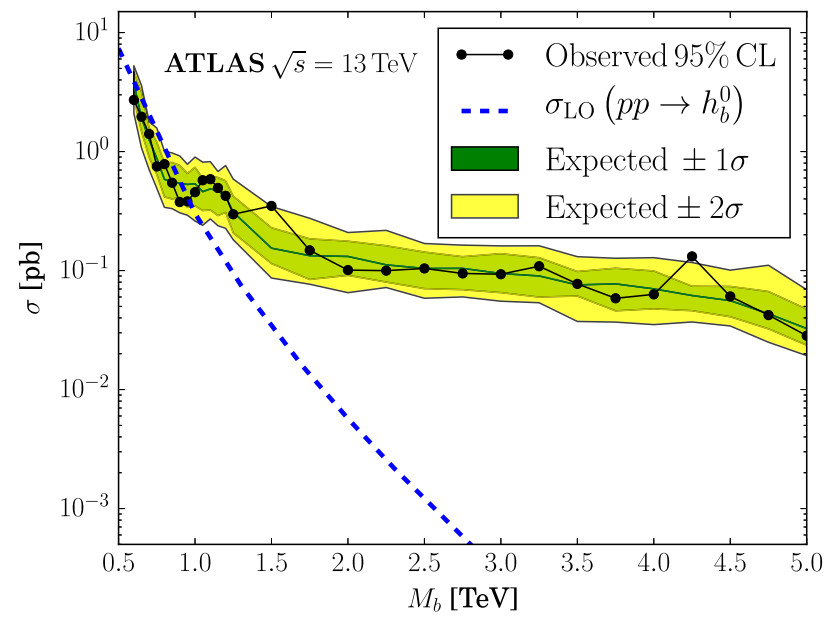

FIG. 2. The green (yellow) region shows the $\pm 1 \sigma( \pm 2 \sigma)$ expected limit for the number of events from $Z^{\prime}$ decaying to two $b$ quarks. We note that the observed cross section (within $95 \%$ C.L) is within $2 \sigma$ of the expected cross section. The blue lines show the predicted LO inclusive cross section of $h_{b}^{0}$ production. The observed and expected data were analyzed by an ATLAS group [28], and these data were made available via HEPDATA [29]. section of $h_{b}^{0}$ was multiplied by a relatively stringent efficiency factor, $\left|\epsilon_{2 b-\mathrm{jet}}\right|=0.2$, to account for the two $-b$ jet reconstruction efficiency. To justify this choice of efficiency, we refer to Ref. [28], which details the $b$ tagging efficiencies for both low and high mass regions. They found for the low-dijet mass regions the efficiency for tagging two $b$ jets decreased from 0.5 to 0.2 as $m_{b b}$ was increased from 0.65 to $1.25 \mathrm{TeV}$. However, in the high-dijet mass region, the event tagging efficiency for two $b$ jets ranged from $0.4-0.05$ for masses as $m_{b b}$ was increased from 1.25 to $5.0 \mathrm{TeV}$.

The total inclusive cross section for charged and neutral Higgs production in heavy-quark annihilation has been calculated at next-to-next-to-leading (NNLO) accuracy in QCD [30]. For masses of new Higgses of $600 \mathrm{GeV}$ (which couple solely to $b$ quarks), the NNLO corrections are small, $\sigma_{\mathrm{NNLO}} / \sigma_{\mathrm{LO}} \sim 0.95$. Moreover, it is likely this $\mathrm{K}$ factor will remain small for larger Higgs masses, and therefore we did not calculate higher-order corrections to the cross section.

From Fig. 2, it seems likely that the LHC could exclude the lower-mass region, $M_{b} \lesssim 1.0 \mathrm{TeV}$, and dedicated studies are encouraged.

\section{B. Prospects for discovery}

In the left plot of Fig. 3, we show the LO production cross section for single $h_{b}^{0}$ production as a function of $h_{b}^{0}$ mass for three c.m. energies: 13, 26, and $100 \mathrm{TeV}$. As masses below $1 \mathrm{TeV}$ are disfavored by data and the mass regime of approximately $3.5 \mathrm{TeV}$ is favored theoretically, we shall discuss the latter. For masses $M_{b}=3.5 \mathrm{TeV}$, the corresponding cross section is approximately $2.4 \times 10^{-4}$, 0.011 and $2.0 \mathrm{pb}$ for $\sqrt{s}=13,26$, and $100 \mathrm{TeV}$, respectively. In the right plot of Fig. 3, we display the LO cross section as a function of c.m. energy for three fixed masses $M_{b}=1.0,2.0,3.5 \mathrm{TeV}$ as shown in purple, green, and red, respectively. Naturally, the cross section increases for higher c.m. energies.

The most crucial plots in addressing the question of observability are the two plots shown in Fig. 4. The quantity of interest is the significance, defined as $S / \sqrt{B}$, where $S$ is the expected number of signal counts and $B$ is the expected number of background (BG) counts. The left and right plots display the significance, $S / \sqrt{B}$, as a function of integrated luminosity for c.m. energies $\sqrt{s}=13,26 \mathrm{TeV}$, respectively. The significance is defined to be

$$
\frac{S}{\sqrt{B}}=0.5\left(\mathcal{L}\left|\epsilon_{2 b-\mathrm{jet}}\right|\right)^{1 / 2} \frac{\sigma_{\mathrm{LO}}\left(p p \rightarrow h_{b}^{0}\right)}{\left[\sigma_{\mathrm{SM}}(p p \rightarrow b \bar{b})\right]^{1 / 2}},
$$

where $\mathcal{L}$ is the integrated luminosity and $\left|\epsilon_{2 b-\text { jet }}\right|=0.2$ is the applied reconstruction efficiency for two $b$ jets, which we assume is the same for both signal and SM background. 

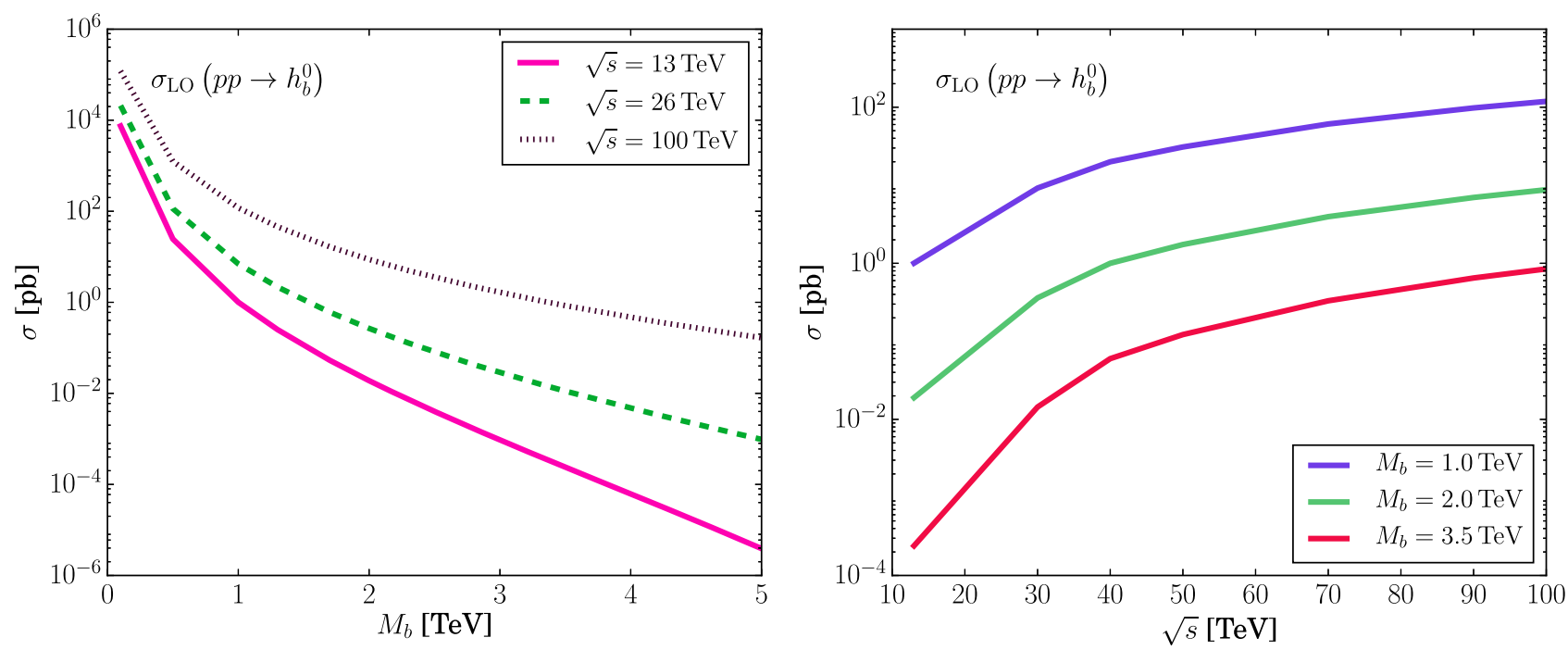

FIG. 3. The left plot displays the total LO cross section for single $h_{b}^{0}$ production as a function of its mass, $M_{b}$, for three fixed c.m. energies $\sqrt{s}=13,26$, and $100 \mathrm{TeV}$ as indicated by the solid fuchsia, dashed green, and dotted purple lines. The right plot shows the LO cross section as a function of c.m. energy for three fixed mass $M_{b}=1.0,2.0,3.5 \mathrm{TeV}$ as indicated by solid purple, green, and red lines.
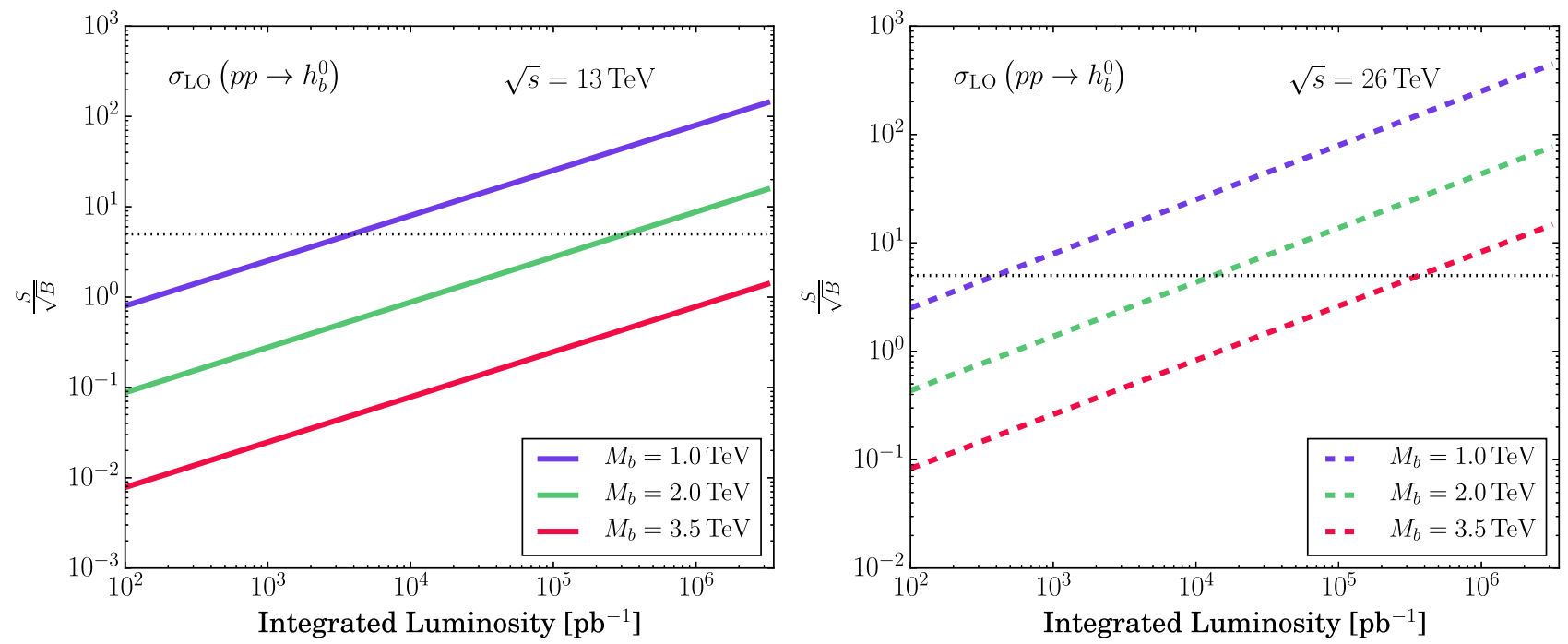

FIG. 4. The left plot displays the significance, $S / \sqrt{B}$, as a function of integrated luminosity at $\sqrt{s}=13$ TeV for three fixed masses $M_{b}=1.0,2.0,3.5 \mathrm{TeV}$ as indicated by solid purple, green, and red. The right plot shows the analogous information but with a c.m. energy, $\sqrt{s}=26 \mathrm{TeV}$.

To estimate the SM background, we applied a number of cuts on the di- $b$ quark production,

(i) $p_{T}(b) \geq 100 \mathrm{GeV}$

(ii) $M_{b}-\Gamma\left(h_{b}^{0}\right) \leq m_{b b} \leq M_{b}+\Gamma\left(h_{b}^{0}\right)$

(iii) $|y|>3.0$

where $p_{T}(b)$ is the transverse momentum of each of the $b$ quarks, $m_{b b}$ is the invariant mass of the $b$-quark system, $\Gamma\left(h_{b}^{0}\right)=3 M_{b} / 8 \pi$ is the width of $h_{b}^{0}$, and $y$ is the rapidity. We note that the cross sections have been calculated at the level of the hard matrix element. The above cuts were applied to the invariant mass system of the two $b$ jets of the BG. We did not apply the cuts to the signal process but have chosen them in such a manner as to not significantly reduce the signal strength. To approximate the effect of the cuts on the signal, we assumed a Breit-Wigner distribution for the differential cross section as a function of the invariant mass such that the chosen range captures approximately half of the signal events. Subsequently, we multiplied the signal by a factor of 0.5, cf., Eq. (39).

In both plots of Fig. 4, the $S / \sqrt{B}$ value is shown for three fixed masses 1.0, 2.0, and $3.5 \mathrm{TeV}$ (as indicated by the purple, green, and red colors, respectively) and at two c.m. energies: 13 and $26 \mathrm{TeV}$ (solid and dashed lines, respectively). The integrated luminosity takes the range between 
$\left(10^{2}-3.5 \times 10^{6}\right) \mathrm{pb}^{-1}$. The value $S / \sqrt{B}=5$ is indicated by the dashed black lines and provides an estimate of the required integrated luminosity for the discovery of $h_{b}^{0}$.

For current c.m. energies, the masses, $M_{b}=1.0$, 2.0 TeV, would be discoverable at integrated luminosities of approximately $2 \times 10^{3}$ and $2 \times 10^{5} \mathrm{pb}^{-1}$, respectively. However, for the heavier mass $M_{b}=3.5 \mathrm{TeV}$, this would require greater than $3.5 \times 10^{6} \mathrm{pb}^{-1}$ of integrated luminosity. With higher c.m. energies, such as $26 \mathrm{TeV}$, the masses $M_{b}=1.0,2.0,3.5 \mathrm{TeV}$ would all become accessible at approximately $5 \times 10^{2}, 10^{4}, 2 \times 10^{5} \mathrm{pb}^{-1}$, respectively. Although we have not shown the projections of the significance for a c.m. collider with $\sqrt{s}=100 \mathrm{TeV}$, we have found that $h_{b}^{0}$ of all three masses can be discovered with less than $3 \mathrm{ab}^{-1}$ of integrated luminosity.

In the event that $h_{b}^{0}$ were discovered, a search and discovery of its charged counterpart, $h_{b}^{ \pm}$, would complete the doublet, $H_{b}$. Although, $h_{b}^{ \pm}$is mass degenerate with $h_{b}^{0}$, its production cross section is smaller by at least an order of magnitude because it couples to $\bar{t} b$ or $\bar{b} t$ and therefore cannot be produced from heavy-quark fusion. The leading production channel of $h_{b}^{ \pm}$is in association with a bottom and a top quark, in which the final state will be two $b$ jets and two further jets. Such a search would be more feasible than di- $h_{b}^{ \pm}$production, which is mainly mediated via photons or an off-shell $\mathrm{Z}$ boson, as this process is kinematically suppressed from the two Higgses in the final state.

One could also consider the production of the lightest dormant doublet associated to the lepton sector, $H_{\tau}$. Because of its large coupling to $\tau, g_{\ell}=0.7$, the neutral $h_{\tau}^{0}$ decays dominantly to $\tau \tau$. As $H_{\tau}$ does not couple strongly to quarks, it can only be produced via electroweak interactions, making it challenging to probe at the LHC. In spite of the fact the $\tau$ reconstruction efficiency is slightly higher than that of $b$ quarks $\left(\left|\epsilon_{\tau}\right| \sim 0.8\right.$ as measured at $\sqrt{s}=13 \mathrm{TeV}$ [31]), as the $h_{\tau}^{0}$ production is electroweak in nature, the cross section is significantly (several orders of magnitude) suppressed compared with the analogous production of $h_{b}^{0}$.

The production and detection and integrated luminosities required for $5 \sigma$ discovery are also discussed in Ref. [12]. Although this discussion has been largely schematic, and certainly an in-depth analysis would be needed to search for the lightest dormant Higgs of the scalar democracy, we find it an encouraging first step in demonstrating that the lightest dormant Higgs is within reach at current energies and most certainly future upgrades.

\section{Meson-antimeson mixing}

Having discussed the main production channels for the dormant Higgses, we now turn to the implications of the scalar democracy for flavor phenomenology in the simple limit discussed in Sec. III B, in which the dormant Higgses do not mix with each other. The NP parameters are reduced from several hundreds in the generic model down to a more manageable set consisting of the dormant Higgs masses and the fermion rotation matrices, $\mathcal{L}_{f}, \mathcal{R}_{f}$, once we impose the requirement that SM fermion masses must be reproduced.

Potentially large FCNCs may arise when the quark fields are rotated from their gauge to their mass eigenbasis and will place stringent constraints on the mass scale of these dormant Higgses. The constraints from flavor-violating processes in both the quark and lepton sectors have been studied in depth in the context of two Higgs doublet models; see, e.g., Ref. [32].

Integrating out the heavy Higgs mass eigenstates gives NP contributions to the effective four-fermion operators at the low-energy scale relevant for flavor physics. The neutral Higgses induce new four-fermion operators already at tree level, contributing to the effective low-energy interactions among fermions with flavor indices $(q, p, r, s)$, namely,

$$
\mathcal{L}_{\text {eff }} \supset-\sum_{f, i j \neq u, 33} \frac{g^{2}}{\left(M_{i j}^{f}\right)^{2}} \mathcal{L}_{f, q i} \mathcal{L}_{f, s i}^{*} \mathcal{R}_{f, p j}^{*} \mathcal{R}_{f, r j}\left(\bar{f}_{\mathrm{L}}^{q} f_{\mathrm{R}}^{p}\right)\left(\bar{f}_{\mathrm{R}}^{r} f_{\mathrm{L}}^{s}\right)
$$

The sum runs over all fermions except the $t$ quark denoted by $(u, 33)$, in order to avoid double counting, as this would be the SMH contribution. Besides, the SMH couplings are aligned with the fermion masses and thus do not lead to tree-level flavor-changing transitions. The tree-level exchange is in most cases expected to be the leading NP contribution to the $\Delta F=2$ operators, as it is only suppressed by the mass of the dormant Higgses mediating the process.

The right-handed rotation matrices, $\mathcal{R}_{f}$, are not constrained a priori as they are unphysical in the SM. The limit $\mathcal{R}_{f}=\mathbb{1}_{3}$ is thus viable and turns off the $\Delta F=2$ operators in Eq. (40), as discussed in Sec. III C. Near this limit, the leading NP contributions will not come from tree-level contributions but will rather be induced at the one-loop level from box diagrams with neutral or charged Higgses with or without SM charged currents $W$ bosons.

We will focus on meson-antimeson mixing, which is $\Delta F=2$ observables that are loop and GIM suppressed in the SM. These observables are thus sensitive to NP degrees of freedom, which propagate in the mixing amplitudes. As such, signals of NP may be constrained through comparison of observed meson mixing and the SM predictions.

Meson mixing has been observed in the $D^{0}-\bar{D}^{0}, B_{s}-\bar{B}_{s}$, $B^{0}-\bar{B}^{0}$, and $K^{0}-\bar{K}^{0}$ systems. In each case, the observed mass splitting, $\Delta M$, of the resulting meson mass eigenstates constrains the magnitude of the transition amplitudes. Although the experimental values are known to high precision [33], the SM theory predictions are plagued by large hadronic uncertainties, and there is still room for 
TABLE II. Bounds used to constrain the size of the NP contributions to FCNC operators relevant for meson-antimeson mixing [24].

\begin{tabular}{lcc}
\hline \hline & \multicolumn{2}{c}{ Bounds on $C\left(\mathrm{TeV}^{-2}\right)$} \\
\cline { 2 - 3 } Operator & $\mathrm{Re}$ & $\mathrm{Im}$ \\
\hline$C\left(\bar{s}_{\mathrm{R}} d_{\mathrm{L}}\right)\left(\bar{s}_{\mathrm{L}} d_{\mathrm{R}}\right)$ & $6.9 \times 10^{-9}$ & $2.6 \times 10^{-11}$ \\
$C\left(\bar{b}_{\mathrm{R}} d_{\mathrm{L}}\right)\left(\bar{b}_{\mathrm{L}} d_{\mathrm{R}}\right)$ & $3.9 \times 10^{-7}$ & $1.7 \times 10^{-7}$ \\
$C\left(\bar{b}_{\mathrm{R}} s_{\mathrm{L}}\right)\left(\bar{b}_{\mathrm{L}} s_{\mathrm{R}}\right)$ & $8.8 \times 10^{-6}$ & $2.9 \times 10^{-6}$ \\
$C\left(\bar{c}_{\mathrm{R}} u_{\mathrm{L}}\right)\left(\bar{c}_{\mathrm{L}} u_{\mathrm{R}}\right)$ & $5.7 \times 10^{-8}$ & $1.1 \times 10^{-8}$ \\
\hline \hline
\end{tabular}

NP [34,35]. The phases of the transition amplitudes give rise to $C P$ violation in the respective meson-antimeson systems as observed through the parameters $\phi_{D}, \epsilon_{K}, S_{\psi K_{s}}$, and $S_{\psi \phi}$.

An exact analysis of the flavor constraints is somewhat beyond the scope of this paper. We will content ourselves with a rough estimate for the allowed mass range for each of the dormant Higgses. To this end, we apply the bounds of Isidori [24] on the Wilson coefficients for the scalar exchange effective operators of Eq. (40), cf., Table II.

To assess the parameter space, we will probe the NP constraints using a number of benchmark cases for the rotation matrices. In each case, flavor bounds will place a lower limit on the masses of the dormant Higgses, and we will compare these to the expected masses obtained from the fine-tuning arguments, cf., Eq. (32).

The meson-antimeson mixing bounds on NP contribution to the $\Delta F=2$ operators can be translated into bounds on the dormant Higgs masses once $\mathcal{L}_{q}$ and $\mathcal{R}_{q}$ are assumed. For each Higgs, we determine the mass at which it saturates each of the bounds in the absence of contribution from other dormant Higgses; the highest such mass is used as a lower bound for the corresponding Higgs mass. This is a somewhat simplified approach to the constraints, as it discounts possible interference between contributions from multiple Higgses, not to mention the running of the effective operators below the EW scale. Since we are only exploring a small region of parameter space of the full model in any case, the bounds we obtain using these approximations remain a good indication for the scale in which the Higgses become visible in flavor physics.

The benchmark points we consider are informed by the constraint that the left-handed quark mixings must reproduce the CKM matrix. Hence, it is unavoidable to have some left-handed mixing, albeit there is still freedom to put all of the left-handed mixing into either the up or the down sector. Good middle ground would seem to be taking a half-and-half approach. One will quickly find that with this assumption for the left-handed rotation the meson-antimeson mixing constraints will favor small mixing of the right-handed quarks. We consider the following benchmarks:
(1) $\mathcal{L}_{u}=\mathcal{L}_{d}^{\dagger}=V_{\mathrm{CKM}}^{1 / 2}, \quad \mathcal{R}_{u}^{\dagger}=\mathcal{R}_{d}=\mathbb{1}_{3}$
(2) $\mathcal{L}_{u}=\mathcal{L}_{d}^{\dagger}=V_{\mathrm{CKM}}^{1 / 2}, \quad \mathcal{R}_{u}=\mathcal{R}_{d}=V(1,1)$
(3) $\mathcal{L}_{u}=\mathcal{L}_{d}^{\dagger}=V_{\mathrm{CKM}}^{1 / 2}, \quad \mathcal{R}_{u}=\mathcal{R}_{d}=V(3,3)$
(4) $\mathcal{L}_{u}=\mathcal{L}_{d}^{\dagger}=V_{\mathrm{CKM}}^{1 / 2}, \quad \mathcal{R}_{u}^{\dagger}=\mathcal{R}_{d}=V_{\mathrm{CKM}}^{1 / 3000}$
(5) $\mathcal{L}_{u}=\mathcal{L}_{d}^{\dagger}=V_{\mathrm{CKM}}^{1 / 2}, \quad \mathcal{R}_{u}^{\dagger}=\mathcal{R}_{d}=V_{\mathrm{CKM}}^{1 / 30}$
(6) $\mathcal{L}_{u}=\mathbb{1}_{3}, \quad \mathcal{L}_{d}^{\dagger}=V_{\mathrm{CKM}}$,
$\mathcal{R}_{u}^{\dagger}=V_{\mathrm{CKM}}^{1 / 2}, \quad \mathcal{R}_{d}=V_{\mathrm{CKM}}^{1 / 300}$,

where

$V\left(\eta_{1}, \eta_{2}\right)=\left(\begin{array}{ccc}1 & \eta_{1} \times 10^{-4} & 0 \\ -\eta_{1} \times 10^{-4} & 1 & 8 \eta_{2} \times 10^{-3} \\ 0 & -8 \eta_{2} \times 10^{-3} & 1\end{array}\right)$

is an approximately unitary matrix. For each benchmark, we have determined the lower bound individually for each dormant Higgs mass, for all the effective operators in Table II to satisfy their bounds. The resulting lower bounds are plotted in Fig. 5. As a comparison, the gray lines in the figure show the expected masses of the Higgses assuming the absence of fine-tuning, cf., Eq. (32). We proceed to investigate the benchmark points one at a time.

Benchmark 1 shows the mass spectrum of the theory in the absence of right-handed rotation. The Higgs mass estimates in Fig. 5 exhibit the inverse hierarchy of the quark masses detailed in Table I with the Higgses associated to the lighter quarks tending to be the heaviest. The nontrivial left-handed rotation will lower the mass estimates for the off-diagonal mass. In particular, $H_{c t}$ must be almost as light as $H_{b}$ in order to generate a large offdiagonal mass element in the fermion mass matrix. The corresponding plot in Fig. 5 does not show any bounds on the Higgs masses, as we have not included the loopinduced effects here.

Benchmarks 2 and 3 illustrate a marginal case for how much right-handed rotation can be allowed without tension between mass estimates and flavor bounds. The matrix $V\left(\eta_{1}, \eta_{2}\right)$ used to parametrize the right-handed matrix is applied for both $\mathcal{R}_{u}$ and $\mathcal{R}_{d}$ for simplicity. The $(1,2)$ entry governs the mixing of the first two generations of quarks. As $\eta_{1}$ is increased so are the flavor bounds on $H_{u}, H_{u c}$, $H_{c u}$, and $H_{c}$, because they are governed by $D^{0}$ mixing (between the $u$ and $c$ quarks). Similarly, the bounds on the $H_{d}, H_{d s}, H_{s d}$, and $H_{s}$ from $K^{0}$ are pushed up, though they are less problematic. The entry $(1,3)$ of $V\left(\eta_{1}, \eta_{2}\right)$ governs the bound on $H_{b}$ from $B_{s}$ mixing in a similar manner. Going from the marginal case $\eta_{1}=\eta_{2}=1$ in benchmark 2 to the case $\eta_{1}=\eta_{2}=3$ in benchmark 3 , we observe how all the corresponding flavor bounds are increased by a 


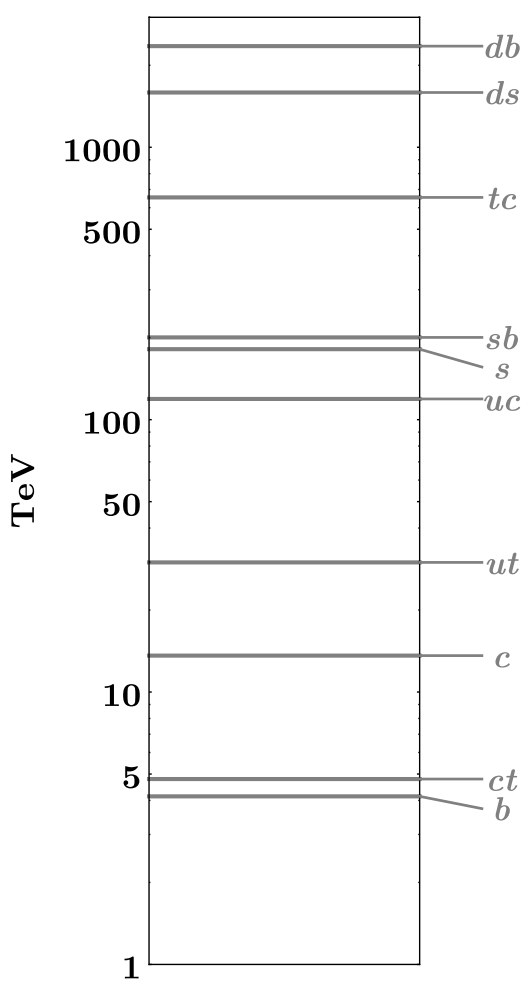

Benchmark 1

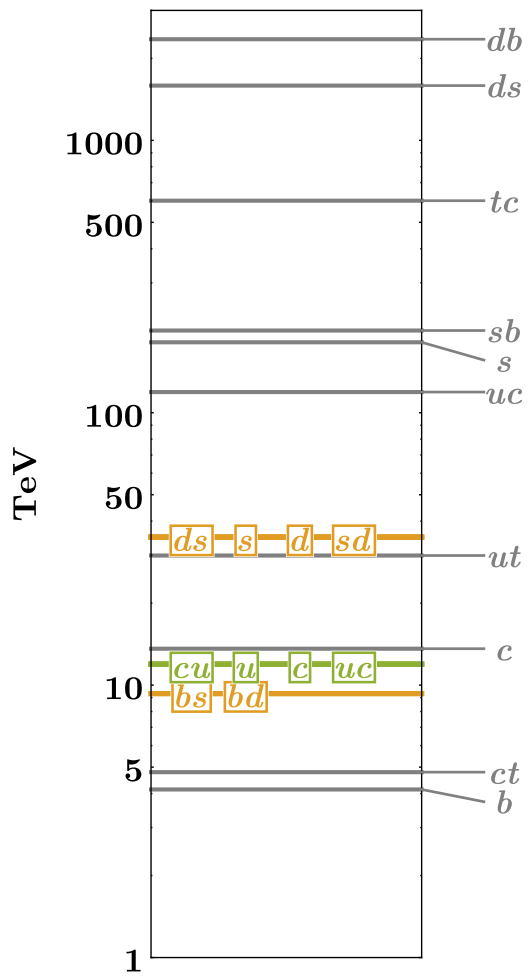

Benchmark 4

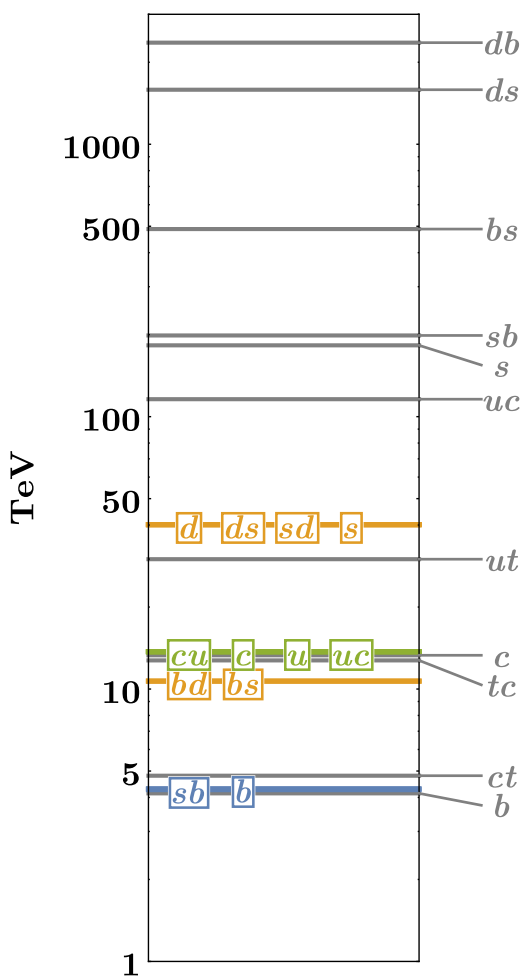

Benchmark 2

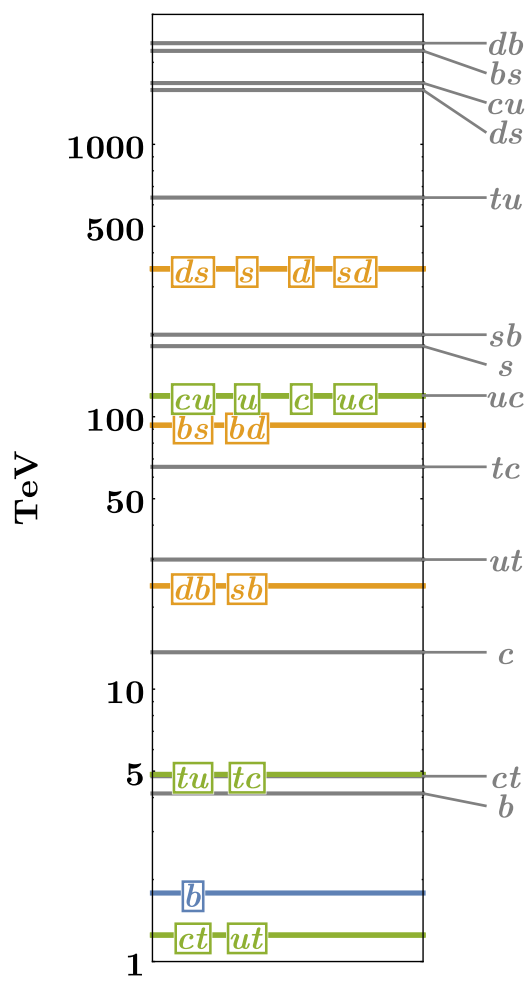

Benchmark 5

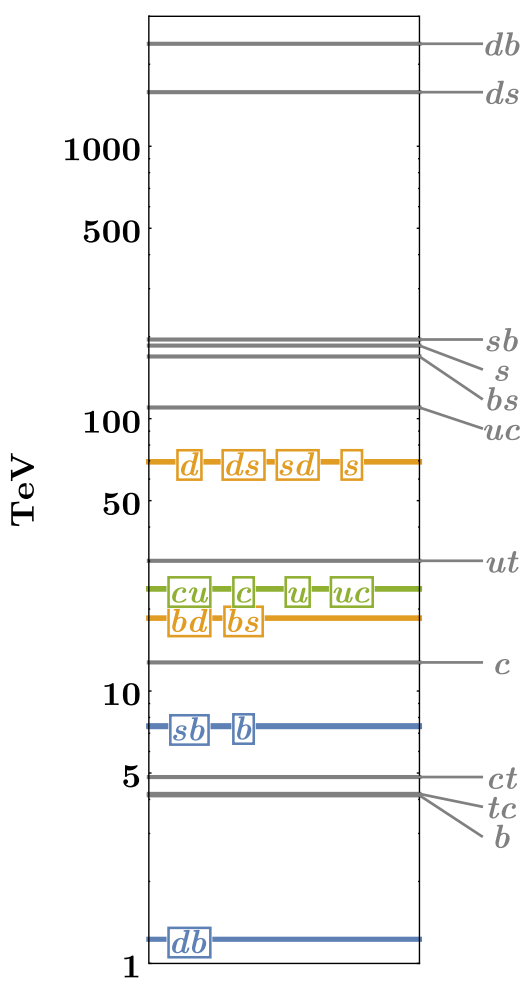

Benchmark 3

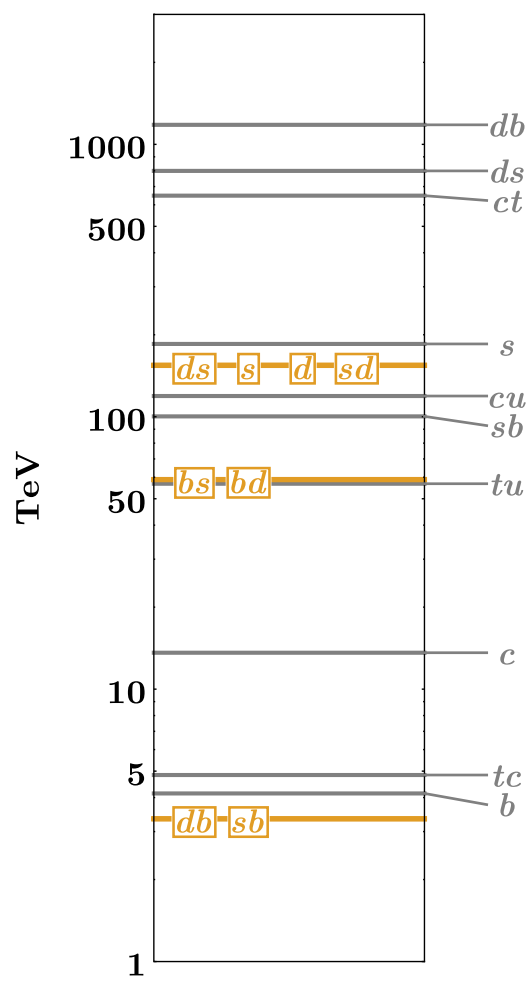

Benchmark 6

FIG. 5. Experimental constraints and mass estimates for the dormant Higgses in the quark sector in six different benchmarks (see the text); the labels denote the indices of the corresponding Higgs. The gray lines are the mass estimates. The colored lines correspond to the most stringent experimental lower bound on each of the Higgs masses: orange if the constraint is from $D_{0}$ mixing, green from $K_{0}$, and blue from $B_{s}$. If a mass-estimate entry is not shown, it is above the scale of the plot. Similarly, if a mass bound is not shown, it is below the scale of the plot. 
TABLE III. Dormant Higgs bosons masses, for different neutrino mass generation mechanisms, assuming (1) the level-repulsion feedback on the Higgs is limited to $(100 \mathrm{GeV})^{2}$ for each neutrino, and (2) $\mu=100 \mathrm{GeV}$ for all mixings (see text for details). Here, $v=175 \mathrm{GeV}$ and $g_{\ell}=0.7$. The requirement of a minimum mass for the heaviest neutrino typically translates into a low bound on the mass of some scalar.

\begin{tabular}{lcccc}
\hline \hline Scenario & Higgs field & Neutrino mass & Case (1) & Case (2) \\
\hline Dirac & $H_{\nu}=v \frac{\mu^{2}}{M_{\nu}^{2}}+H_{\nu}^{\prime}$ & $m_{\nu}=g_{\ell} \frac{\mu^{2}}{M_{\nu}}<0.06 \mathrm{eV}$ & $M_{\nu}>2 \times 10^{14} \mathrm{GeV}$ & $M_{\nu}>1.4 \times 10^{8} \mathrm{GeV}$ \\
& $m_{\nu}^{\text {heaviest }}>0.05 \mathrm{eV}$ & $M_{\nu}^{\text {lightest }}<2.4 \times 10^{14} \mathrm{GeV}$ & $M_{\nu}^{\text {lightest }}<1.5 \times 10^{8} \mathrm{GeV}$ \\
Type-I & $S_{N}=\mu \frac{v^{2}}{M_{S_{N}}^{2}}+S_{N}^{\prime}$ & $m_{N}=g_{\ell} \mu \frac{v^{2}}{M_{S_{N}}^{2}}$ & No sharp prediction & see Fig. 6 \\
Type-II & $\Delta=\mu \frac{\nu^{2}}{M_{\Delta}^{2}}+\Delta^{\prime}$ & $m_{\nu}=g_{\ell} \mu \frac{v^{2}}{M_{\Delta}^{2}}<0.06 \mathrm{eV}$ & $M_{\Delta}>2 \times 10^{14} \mathrm{GeV}$ & $M_{\Delta}>1.8 \times 10^{8} \mathrm{GeV}$ \\
& $m_{\nu}^{\text {heaviest }}>0.05 \mathrm{eV}$ & $M_{\Delta}^{\text {lightest }}<2.4 \times 10^{14} \mathrm{GeV}$ & $M_{\Delta}^{\text {lightest }}<2 \times 10^{8} \mathrm{GeV}$ \\
\hline \hline
\end{tabular}

factor $\sqrt{3}$, giving slight tension between mass estimates and flavor bounds. We would like to emphasize that this tension only exists in the complete absence of fine-tuning of the Higgs mass, $-M_{0}^{2}$. If one were to tolerate fine-tuning to the percent level, the Higgs mass estimates would increase by a factor 10 .

Benchmarks 4 and 5 parametrize $\mathcal{R}_{q}$ in terms of powers of the CKM matrix to illustrate how large the right-handed mixings are allowed to be in terms of a more familiar matrix. Because of the relatively large mixing, $\left(V_{\mathrm{CKM}}\right)_{12} \simeq\left(V_{\mathrm{CKM}}\right)_{21} \simeq 0.23$, one has to go to the case $\mathcal{R}_{u}^{\dagger}=\mathcal{R}_{d}=V_{\text {CKM }}^{1 / 3000}$ of benchmark 4 , before the flavor bounds decrease below the mass estimates of $H_{c}$. On the other hand, if one is willing to accept a percent-level fine-tuning of the Higgs mass, we can go to the case $\mathcal{R}_{u}^{\dagger}=$ $\mathcal{R}_{d}=V_{\text {CKM }}^{1 / 30}$ of benchmark 5 . We stress that the mass estimates shown in Fig. 5 are all with the complete absence of fine-tuning.

Benchmark 6 puts the entire left-handed rotation into $\mathcal{L}_{d}$ and gives an alternative example of evading the flavor bounds. In this case, a large $\mathcal{R}_{u}$ can be tolerated without generating $D^{0}$ mixing at tree level. The main constraints in this case are from $K^{0}$ mixing.

Though our selection of a few benchmark points does not constitute an in-depth analysis of the flavor physics of the scalar democracy, it demonstrates that there is room for the framework to avoid tension with flavor physics. Yet, care has to be taken to ensure that one works in an allowed regions of parameter space.

\section{LEPTON-SECTOR PHENOMENOLOGY}

\section{A. Neutrino sector}

In our framework, the mass spectrum of SM fermions is mapped onto a scalar mass spectrum in which the lighter scalars develop larger VEVs and thus leads to heavier fermion masses; see Eq. (32). As the masses of charged fermions have been precisely measured, a sharper prediction of the scalar spectrum can be made. We do not know the absolute value of neutrino masses nor the mass generation mechanism, resulting in looser possibilities for the scalar bound states associated to neutrino masses. The goal of this section is to examine these possibilities.

Although the absolute values of neutrino masses are still to be measured, neutrino oscillations give us valuable information on their masses. From the KamLAND experiment and observations of the solar neutrino spectrum $[36,37]$, the solar mass splitting ${ }^{9}$ has been measured to be $\Delta m_{21}^{2} \equiv m_{2}^{2}-m_{1}^{2} \simeq+7.4 \times 10^{-5} \mathrm{eV}^{2}$. The sign of $\Delta m_{21}^{2}$ is known due to matter potential effects on neutrino oscillations. Besides, atmospheric and accelerator neutrino oscillation experiments [38-40] are compatible with $\left|\Delta m_{31}^{2}\right| \equiv\left|m_{3}^{2}-m_{1}^{2}\right| \simeq 2.5 \times 10^{-3} \mathrm{eV}^{2}$. Therefore, the following two neutrino mass orderings are still viable experimentally, $m_{1}<m_{2}<m_{3}$ or $m_{3}<m_{1}<m_{2}$ (recent experiments exhibit a slight preference toward the former scenario [41,42]). In addition, the sum of neutrino masses is bounded from above by $0.12 \mathrm{eV}$ from cosmological observations [43]. We can thus identify three extreme scenarios for the neutrino mass spectrum $\left(m_{1}, m_{2}, m_{3}\right)$ : normal hierarchy $(0,0.008 \mathrm{eV}, 0.05 \mathrm{eV})$, inverted hierarchy (0.05 eV, $0.05 \mathrm{eV}, 0)$, and semidegenerate neutrinos approximately $(0.03 \mathrm{eV}, 0.03 \mathrm{eV}, 0.06 \mathrm{eV})$. As the heaviest neutrino needs to have a mass above $0.05 \mathrm{eV}$, that will typically translate into an upper bound for some of the scalars (see Table III).

In the scalar democracy, there are three viable alternatives for the generation of neutrino masses. The neutrino mass generation can be similar to the charged fermion mass generation, as outlined in Eq. (30). In this case, neutrinos would be Dirac fermions. Nevertheless, due to the quantum numbers of the lepton doublet, $L_{\mathrm{L}}$, and the right-handed neutrino, $N_{\mathrm{R}}$, two other bound states can contribute to neutrino masses, namely, $S_{N} \sim \bar{N}_{\mathrm{R}} N_{\mathrm{L}}^{C}$ and $\Delta \sim \bar{L}_{\mathrm{L}} L_{\mathrm{R}}^{C}$. While $H_{\nu}$ may lead to Dirac neutrino masses, an accompanying nonzero expectation value for $S_{N}$ would constitute a Majorana mass term for right-handed neutrinos, realizing a type-I seesaw mechanism, whereas $\Delta$ may

\footnotetext{
${ }^{9}$ The three neutrino mass eigenstates are labeled such that $\nu_{1}$ $\left(\nu_{3}\right)$ has the largest (smallest) admixture of $\nu_{e}$.
} 
be identified as the scalar triplet in type-II seesaw models (see Refs. [44-51]). Thus, the mechanism of neutrino masses depends on which dormant Higgses acquire a VEV.

In the type-I seesaw realization, the relevant terms in the Lagrangian are (generation indices have been suppressed)

$$
\mathcal{L} \supset-g_{\ell} \bar{L}_{\mathrm{L}} \tilde{H}_{\nu} N_{\mathrm{R}}-g_{\ell} S_{N} \bar{N}_{\mathrm{R}} N_{\mathrm{L}}^{C},
$$

and hence neutrino masses are given by

$$
m_{\nu}^{\mathrm{type-}}=g_{\ell} \frac{\left\langle H_{\nu}\right\rangle^{2}}{\left\langle S_{N}\right\rangle}, \quad m_{N}=g_{\ell}\left\langle S_{N}\right\rangle
$$

Because $S_{N}$ is a SM singlet, it will not acquire its VEV from mixing with the $\mathrm{SMH}$ as in Eq. (2) but rather from the term $\mu_{S} H_{0}^{\dagger} H_{0} S_{N}$, which gives $\left\langle S_{N}\right\rangle=\mu_{S} v^{2} / M_{S}^{2}$. Requiring electroweak values for the dimensionful parameter $\mu_{S}=100 \mathrm{GeV}$ and imposing constraints on singletHiggs mixing and active-sterile neutrino mixing lead to the allowed region in the $\left(M_{S}, M_{\nu}\right)$ plane shown in Fig. 6 . The gray regions are excluded due to mixings, while the blue region predicts too-heavy neutrinos. ${ }^{10}$ The atmospheric mass splitting requires at least one neutrino to be heavier than $0.05 \mathrm{eV}$. A dashed black line corresponding to $m_{\nu}=0.01 \mathrm{eV}$ is drawn to guide the reader. Notice that a sort of seesaw mechanism between the sterile neutrino and the singlet scalar is in place: the heavier is $S$, the smaller its VEV, and thus the lighter $N$ is (see the arrows in Fig. 6). If instead we require the level-repulsion feedback to the SMH mass to be small, no sharp prediction can be made about $M_{S}$, as it can always be made arbitrarily large, leading to pseudo-Dirac neutrinos.

In the case of type-II seesaw, in the absence of $N_{R}$, the $\Delta$ would get its VEV from the usual term $\mu_{\Delta} H_{0}^{T} i \sigma_{2} \Delta^{\dagger} H_{0}$. Neutrino masses would be given by

$m_{\nu}^{\mathrm{type}-\mathrm{II}}=0.42 \mathrm{eV}\left(\frac{g_{\ell}}{0.7}\right)\left(\frac{\mu_{\Delta}}{100 \mathrm{GeV}}\right)\left(\frac{10^{8} \mathrm{GeV}}{M_{\Delta}}\right)^{2}$,

such that electroweak values of $\mu_{\Delta}$ would correspond to scalar triplets around the $10^{8} \mathrm{GeV}$ scale, as can be seen in Table III, "Case (2)" column. Restricting the levelrepulsion feedback on the Higgs potential leads to exactly the same mass constraints as in the Dirac neutrino case. Obtaining scalar triplet masses accessible at the LHC, $M_{\Delta} \sim \mathrm{TeV}$ would require $\mu_{\Delta} \sim 10 \mathrm{eV}$, as it happens in usual type-II seesaw scenarios. For the three mechanisms discussed, a summary of the masses of the scalar bound states responsible for the neutrino spectrum can be found in Table III (labeled as "Dirac," "Type I," and "Type II").

\footnotetext{
${ }^{10}$ Note that the constraint on the active-sterile mixing strongly depends on the mass $m_{N}$ and the active flavor [52]. The value chosen here is extremely conservative.
}

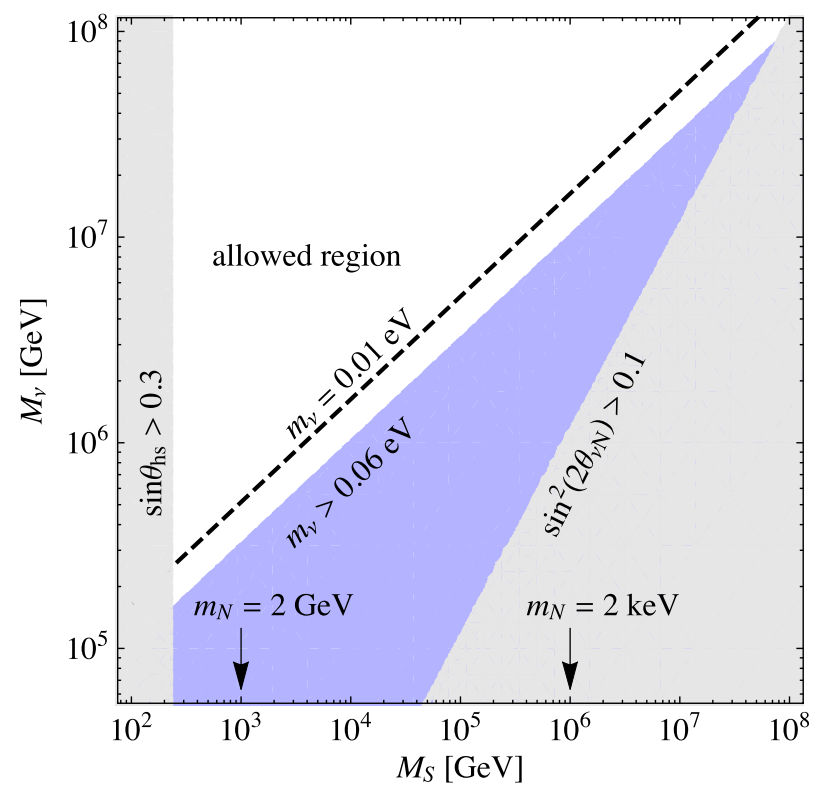

FIG. 6. Mass of scalar singlet $S$ vs scalar doublet $H_{\nu}$ in the scalar democracy type-I seesaw scenario, under the assumption that $\mu_{S}=$ $\mu_{\nu}=100 \mathrm{GeV}$ (see the text for details). The gray regions are ruled out by constraints on singlet-Higgs mixing (leftmost region) and active-sterile neutrino mixing (rightmost region). The blue region predicts too large neutrino masses and is excluded by cosmological observations. The heaviest neutrino is above $0.05 \mathrm{eV}$, and thus at least one pair of scalars should have masses between the dashed black line and the blue region. The mass of the sterile neutrino $m_{N}$ is also indicated for two values of $M_{S}$.

\section{B. Lepton-flavor violation}

Any charged lepton flavor-violating (CLFV) decay would immediately indicate the presence of new physics, and as a consequence, much effort has been put into detecting such effects. The result of this work places severe constraints on the branching ratios for the different CLFV decays of the charged leptons.

In the absence of mixing among dormant Higgses, the CLFV decays must be mediated by the Higgses associated to the lepton mass generation. As a result, the CLFV transitions induced in our framework do not involve quarks. The relevant experimental constraints of this type are the radiative and the three-body decays are shown in Table IV.

The NP contribution to the decay of a charged lepton to another through the emission of a photon is governed, in the low-energy theory, by the dipole operator

$$
\mathcal{L}_{\text {eff }} \supset C_{i j} \bar{E}_{i \mathrm{~L}} \sigma^{\mu \nu} E_{j \mathrm{R}} F^{\mu \nu}+\text { H.c., }
$$

where $i$ and $j$ are flavor indices and $C_{i j}$ has inverse mass dimension. The decay width is given by

$$
\Gamma\left(e_{i} \rightarrow e_{j} \gamma\right)=\frac{m_{e, i}^{3}}{4 \pi}\left(\left|C_{i j}\right|^{2}+\left|C_{j i}\right|^{2}\right) .
$$


TABLE IV. The $90 \%$ C.L. upper limit on decay branching rates for lepton flavor-violating processes.

\begin{tabular}{lc}
\hline $\operatorname{Process}$ & Experimental upper limit \\
\hline $\operatorname{Br}\left(\mu^{+} \rightarrow e^{+} e^{+} e^{-}\right)$ & $1.0 \times 10^{-12}[53]$ \\
$\operatorname{Br}\left(\tau^{-} \rightarrow \mu^{-} \mu^{+} \mu^{-}\right)$ & $2.1 \times 10^{-8}[54]$ \\
$\operatorname{Br}\left(\tau^{-} \rightarrow e^{-} \mu^{+} \mu^{-}\right)$ & $2.7 \times 10^{-8}[54]$ \\
$\operatorname{Br}\left(\tau^{-} \rightarrow \mu^{-} e^{+} e^{-}\right)$ & $1.8 \times 10^{-8}[54]$ \\
$\operatorname{Br}\left(\tau^{-} \rightarrow e^{-} e^{-} e^{+}\right)$ & $2.7 \times 10^{-8}[54]$ \\
$\operatorname{Br}\left(\tau^{-} \rightarrow e^{+} \mu^{-} \mu^{-}\right)$ & $1.7 \times 10^{-8}[54]$ \\
$\operatorname{Br}\left(\tau^{-} \rightarrow \mu^{+} e^{-} e^{-}\right)$ & $1.5 \times 10^{-8}[54]$ \\
$\operatorname{Br}\left(\tau^{ \pm} \rightarrow \mu^{ \pm} \gamma\right)$ & $4.4 \times 10^{-8}[55]$ \\
$\operatorname{Br}\left(\tau^{ \pm} \rightarrow e^{ \pm} \gamma\right)$ & $3.3 \times 10^{-8}[55]$ \\
$\operatorname{Br}\left(\mu^{+} \rightarrow e^{+} \gamma\right)$ & $4.2 \times 10^{-13}[56]$ \\
\hline \hline
\end{tabular}

In the scalar democracy, NP effects are induced by diagrams in which a dormant Higgs propagates in the loop. The coefficients $C_{i j}$ depend only on the rotation matrices of the external fermions and the Higgs mass.

For concreteness, we will assume that the Dirac neutrino mass mechanism is at work (see Table III). The NP contribution to the coefficients of the dipole operators, from both neutral and charged Higgses, is given by

$$
\begin{aligned}
C_{i j}= & \frac{-e g_{\ell}^{2}}{384 \pi^{2}} \sum_{k l}\left(\mathcal{L}_{e, i k} \mathcal{L}_{e, j k}^{*}\left[\frac{2 m_{e, j}}{\left(M_{k l}^{e}\right)^{2}}-\frac{m_{e, j}}{\left(M_{k l}^{\nu}\right)^{2}}\right]\right. \\
& \left.+\mathcal{R}_{e, i k} \mathcal{R}_{e, j k}^{*} \frac{m_{e, i}}{\left(M_{l k}^{e}\right)^{2}}\right),
\end{aligned}
$$

to leading order in $m_{e}^{2} /\left(M^{e}\right)^{2}$, where $M_{k l}^{e}$ denotes the mass of the scalar that generates the $(\mathrm{kl})$ entry of the corresponding leptonic mass matrix. Because of the smallness of neutrino masses, the neutrino Higgs contribution is completely irrelevant to the radiative decay. As an aside, we note that a quick estimate of the NP contribution to $\mu \rightarrow \mu \gamma$ will show that even in the most optimistic scenario $C_{22}$ is several orders of magnitude too small to significantly influence the SM prediction for the muon $g-2$.

The charged lepton decay to three lighter charged leptons is mediated at tree level by neutral Higgses giving the effective operators of Eq. (40). The resulting decay width is

$$
\begin{aligned}
\Gamma\left(e_{p}^{-} \rightarrow e_{r}^{-} e_{s}^{+} e_{t}^{-}\right) \simeq & \frac{7 g_{\ell}^{4} m_{e, p}^{5}}{6144 \pi^{3}}\left(\left|\sum_{i j} \frac{\mathcal{L}_{e, r i} \mathcal{L}_{e, s i}^{*} \mathcal{R}_{e, p j}^{*} \mathcal{R}_{e, t j}}{\left(M_{i j}^{e}\right)^{2}}\right|^{2}\right. \\
& +\left|\sum_{i j} \frac{\mathcal{L}_{e, r i} \mathcal{L}_{e, p i}^{*} \mathcal{R}_{e, s j}^{*} \mathcal{R}_{e, t j}}{\left(M_{i j}^{e}\right)^{2}}\right|^{2} \\
& +(r \leftrightarrow t)) .
\end{aligned}
$$

Many of these decays only violate flavor by one unit, and so, in contrast to the NP contribution to meson-antimeson mixing, the operator does not vanish for all dormant Higgses in the limit where $\mathcal{R}_{e}=\mathbb{1}_{3}$.

Similarly to the quark sector, the only direct constraint on the lepton rotation matrices stem from the requirement $V_{\text {PMNS }}=\mathcal{L}_{\nu} \mathcal{L}_{e}^{\dagger}$. Therefore, we have the same freedom of choice in their parametrization. In contrast to the quark sector, the lepton observables do not significantly favor small rotation angles. For our benchmark points, we have therefore chosen large rotation matrices to illustrate the case in which NP will soon become detectable in LFV observables:

(1) $\mathcal{L}_{e}=\mathcal{R}_{\nu}=V_{\mathrm{PMNS}}^{\dagger 1 / 2}, \quad \mathcal{L}_{\nu}=\mathcal{R}_{e}=V_{\mathrm{PMNS}}^{1 / 2}$

(2) $\mathcal{L}_{e}=\mathcal{R}_{\nu}=U_{\mathrm{TBM}}, \quad \mathcal{L}_{\nu}=\mathcal{R}_{e}=V_{\mathrm{PMNS}} U_{\mathrm{TBM}}$

(3) $\mathcal{L}_{e}=\mathcal{R}_{\nu}=V_{\mathrm{PMNS}}^{\dagger 1 / 2} U_{\mathrm{TBM}}^{1 / 2}, \quad \mathcal{L}_{\nu}=\mathcal{R}_{e}=V_{\mathrm{PMNS}}^{1 / 2} U_{\mathrm{TBM}}^{1 / 2}$.

We apply the PDG convention for the PMNS matrix [57] using the best-fit values of the three mixing angles and phase, $\delta_{\mathrm{CP}}$ [58]. The use of the tribimaximal (TBM) mixing matrix [59-62] is motivated from work on the discrete flavor model, and its structure is given by

$$
U_{\mathrm{TBM}}=\left(\begin{array}{ccc}
-\frac{2}{\sqrt{6}} & \frac{1}{\sqrt{3}} & 0 \\
\frac{1}{\sqrt{6}} & \frac{1}{\sqrt{3}} & \frac{1}{\sqrt{2}} \\
\frac{1}{\sqrt{6}} & \frac{1}{\sqrt{3}} & -\frac{1}{\sqrt{2}}
\end{array}\right) \text {. }
$$

For each of the benchmark points, we have determined the strongest individual limit on each $e$-type dormant Higgs by comparing the NP contribution to the decay bounds in Table IV. We have plotted the leading constraints on the individual Higgs masses resulting from our benchmark points in Fig. 7 together with the corresponding mass estimates resulting from Eq. (32). In the benchmarks, almost all the Higgs mass constraints come from the experimental bounds on the muon decays - even for the dormant Higgses coupling primarily to the third-generation leptons. This is due to the somewhat anarchic structure of the PMNS mixing matrix, meaning that all the Higgses mediate LFV muon decays. The PMNS mixing angle suppression is insufficient to compensate the weaker bounds on the LFV tau branching ratios. Despite the slightly stronger experimental bound on $\operatorname{Br}(\mu \rightarrow e \gamma)$ than $\operatorname{Br}\left(\mu^{-} \rightarrow e^{-} e^{+} e^{-}\right)$, the loop suppression in the leading dormant Higgs contribution to the radiative decay is enough that almost all the $H^{e}$ Higgses get their strongest bound from the decay to three charged leptons. For at least some of the Higgses, it is possible to engineer the rotation matrices in such a way as to avoid the experimental bound on $\operatorname{Br}\left(\mu^{-} \rightarrow e^{-} e^{+} e^{-}\right)$.

Regardless of the anarchic flavor structure chosen for the benchmark points, Fig. 7 shows that there is only severe tension between the mass estimates of $H_{e}, H_{\mu}, H_{e \mu}$, and $H_{\mu e}$ and their corresponding constraints in benchmark 2. 

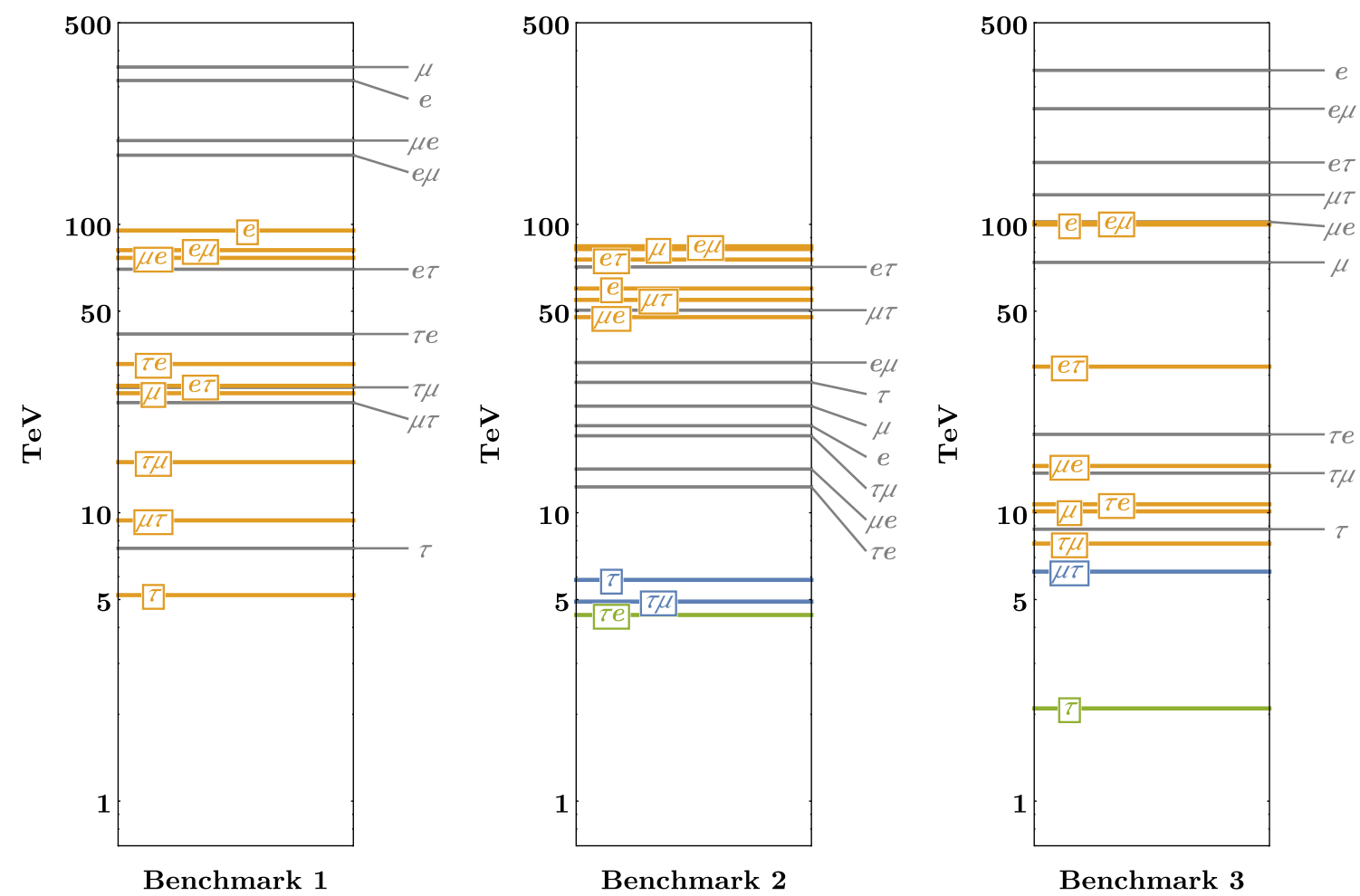

FIG. 7. Experimental constraints and mass estimates for the dormant Higgses in the lepton sector in three different benchmarks; the labels denote the indices of the corresponding Higgs. The gray lines are the mass estimates. The colored lines correspond to the most stringent experimental constraint on each of the Higgs masses: orange if the constraint is from $\mu \rightarrow 3 e$ decays, blue if the constraint is from $\tau \rightarrow 3 \ell$, and green if the constraint is from $\mu \rightarrow e \gamma$.

We therefore expect that most of the parameter space (consistent with our assumptions) will pass the leptonflavor constraints. We also anticipate that future CLFV experiments, with significantly improved sensitivities, will be important probes of this scenario.

The Higgses associated with neutrinos in the Dirac mass scenario, $H^{\nu}$, are only constrained by the radiative decays. In no case, regardless of the left-handed mixing, are the bounds on the $H^{\nu}$ masses stronger than $5 \mathrm{TeV}$, much below what is expected from the fine-tuning argument. For this reason, the corresponding constraints have not been displayed in Fig. 7.

\section{CONCLUSIONS}

In general, we are proposing that a plethora of new scalar bosons exists in nature. To introduce a governing principle, we have argued schematically that every fermion pair binds to form a bound-state scalar boson, due to a universal attractive interaction at a very high scale, $\Lambda$. Among many new states, including leptoquarks, colored isodoublets and singlets, etc., this hypothesis implies the existence of a large number of sequentially more massive Higgs bosons. This is essentially a hypothesis of universal Higgs-Yukawa couplings.

We argue that the SM Higgs boson is the first of the sequence, and therefore must have a dynamical origin, and essentially emerges as a $\bar{t} t$ bound state but is now part of the constellation of composite scalars that influence its mass. One immediate intrigue is that the top-quark mass, predicted by the renormalization group fixed point [20,21], which is the prediction of top condensation models [7], now comes within a few percent of the observed value. This is expected to be improved by including the multi-Higgs boson masses and decouplings and is a first indicator that this could be a pathway to new UV physics.

A universal binding like we invoke may be intimately associated with gravity, since strong scattering amplitudes that are parametrized by $d=6$ operators and scale as $1 / M_{\text {Planck }}^{2}$ may exist near the Planck scale. Among these amplitudes, we would expected a general four-fermion structure such as $\left(g^{2} / M^{2}\right)\left(\bar{\psi}_{\mathrm{L}}^{i} \psi_{\mathrm{R}}^{j}\right)\left(\bar{\psi}_{\mathrm{R}}^{i} \psi_{\mathrm{L}}^{j}\right)$, where $i, j$ run over the conventional quark and lepton flavors and colors of the SM. In fact, since gravity does not distinguish between a particle and antiparticle, we would expect similar operators with a Majorana-like structure. The operators must be gauge invariant, and with the usual SM fields, this contains a subsector of a global $\mathrm{SU}(48) \times \mathrm{SU}(48) \times \mathrm{U}(1)$ chiral Lagrangian. This could also arise from a new strong gauge interaction.

We therefore argue that there exists a sequence of composite Higgs doublets leading upward to large mass scales. These are dormant isodoublets, each having only 
small tadpole VEVs that arise from mixing with the observed Higgs and that scale as $1 / M^{2}$ for heavy Higgs fields of mass $M$. The masses of these scalars are lacking a theory-we view them at present as soft symmetry breaking, relevant operators that we insert by hand in accord with known phenomenology. Nonetheless, the virtue of this model is that the Yukawa interactions are "known" and all are of order unity. The observed spectrum of quarks and leptons and their mixing angles can be codified in terms of the mass-mixing problem in the extended Higgs sector.

Most interesting is the top-bottom system. The top mass and the SM Higgs calibrate the overall Yukawa coupling for all quarks, $g=g_{\text {top }} \simeq 1$. Here, the first sequential dormant Higgs $H_{b}$ couples essentially to $\bar{b} b$ and has a positive mass, $M_{b}^{2}$. It has a mixing with the lightest Higgs $H_{0}$ which causes it to acquire a small VEV that feeds the $b$ quark its mass. We can view the SM Higgs as having initially positive $M_{0}^{2}$ (or zero), and the effect of "level repulsion" by $H_{b}$ drives it to become tachyonic. To avoid significant fine-tuning, we would require $\mu^{4} / M_{b}^{2} \sim M_{H}^{2}$, which leads to a soft "naturalness" upper limit on $M_{H_{b}} \lesssim\left(v_{\text {weak }} / m_{b}\right)(\sim 100 \mathrm{GeV}) \sim$ 3.5 TeV. Note that we have subsequently given a dedicated analysis of the third generation in Ref. [12], which could serve as a stand-alone model with significantly fewer degrees of freedom than the full scalar democracy.

We discuss in some detail a particular search strategy to address the neutral component of $H_{b}=\left(h_{b}^{+}, h_{b}^{0}\right)$, through the process $p p \rightarrow h_{b}^{0} \rightarrow \bar{b} b$. This is available at the current LHC energy and luminosity, and possible upgrades. We emphasize that the LHC already has the capability of excluding sequential $H_{b}$ with $\mathcal{O}(1)$ Yukawa coupling to $\bar{b} b$ in a mass range of order less than $1 \mathrm{TeV}$ (as in the model of Ref. [5]). The full range of $H_{b}$ masses may be accessible to the high luminosity and/or energy-doubled LHC and should help justify the case for such future machines. Our estimates are preliminary and likely will bound above what can be done by improved detector based studies and more detailed deployment of cuts and search strategies. We strongly encourage our experimental colleagues to pursue this. We thus defer details of possible searches $h_{b}^{+} \rightarrow t \bar{b}$ and $H_{\tau}$ elsewhere.

The challenge to scalar democracy is to avoid unwanted $d>4$ flavor transition operators. The rare processes we focus on are mainly neutral meson mixing, and we find that such processes are binding on the Higgs spectroscopy. However, avoiding these constraints in this model is possible since most of the new sequential Higgs bosons are very heavy. Conceivably, though we have not explored it, the extended Higgs sector may provide sources of observable rare processes in heavy meson decays. Discovery of the lighter doublets could thus provide the impetus for partially unraveling the flavor problem.

We also discuss the leptons. Our model most naturally leads to a "neutrino Higgs seesaw" for neutrino Dirac masses in analogy to the quark-sector Higgs structure. However, we have additional fermionic bound states that can, in principle, mix to develop tadpole VEVs and produce Majorana masses, realizing a type-I or type-II seesaw mechanism. Hence, the double- $\beta$ decay experiments will be important probes of the far UV Higgsology of this framework. Our model can also drive rare lepton numberviolation experiments, and we focus discussion on a subset of these, $\ell_{i} \rightarrow \ell_{j}+\gamma$ and $\ell \rightarrow 3 \ell^{\prime}$. We certainly do not exclude other possible processes as probes of the leptonic Higgs system.

One view of the future evolution of fundamental physics with the energy scale argues that the couplings are small and asymptotically free and that the theory "fades away" into a kind of linear scale invariance. We have arrived at a contrary point of view, i.e., that a rich spectrum of new scalar states lies immediately beyond current energy scales and is within reach of the LHC and its upgrade path. In part, this is motivated by chirality; it is very unlikely that one can generate a small Yukawa coupling constant from zero (certainly not perturbatively). Hence, the tiny $g_{\text {electron }} \sim$ $10^{-6}$ is most likely due to a power-law suppression of a coupling that is of order unity, such as $g_{\text {electron }} \sim g_{\ell} \mu_{e}^{2} / M_{e}^{2}$, where $g_{\ell} \sim 1$. The power-law suppression then demands new mass scales, such as $\mu$ and $M$, as realized in our model.

We have argued that a natural way to achieve this "democratically" throughout the entire flavor system of the SM is with a grand enlargement of the scalar system. This flips the flavor problem: the lightest (heaviest) fermions are coupled to the heaviest (lightest) scalars. The resulting spectrum of fermions is due to the inverted spectrum of associated scalars. This focuses urgent attention to the top-bottom (and perhaps the $\tau-\nu_{\tau}$ ) subsystem and is testable at the LHC and future upgrades. We urge our experimental colleagues and other theorists to take up the cause.

While scalar democracy lacks a detailed theoretical underpinning, it provides a first pass-through scenario for a rich spectrum of Higgs, and other scalar, bosons. Many of the issues we describe here would be relevant to large Higgs extensions of the standard model. If some subset of the predictions is confirmed, such as the observation of $H_{b}$, and possibly $H_{\tau}$, with expected $\mathcal{O}(1)$ Yukawa couplings, it would significantly reshape our view of the UV completion of the standard model.

\section{ACKNOWLEDGMENTS}

We thank John Campbell, Andre de Gouvea, Roni Harnik, Josh Isaacson, and Tobias Neumann for helpful discussions and Bogdan Dobrescu and Matthew Low for reading the preliminary draft. One of us recalls a conversation with Bjorken, in which he noticed a plethora of Higgs bosons arising in conjunction with extensions of gravity and which helped to inspire this work [1]. 
This work was done at Fermilab, operated by Fermi Research Alliance, LLC, under Contract No. DE-AC0207CH11359 with the United States Department of Energy. A. E. T. would like to thank Fermilab for hosting him during the writing of this paper and gratefully acknowledges financial support from the Danish Ministry of Higher Education and Science through an EliteForsk Travel Grant. The $\mathrm{CP}^{3}$-Origins center is partially funded by the Danish National Research Foundation, Grant No. DNRF90.
[1] J. D. Bjorken (private communication).

[2] N. V. Krasnikov, Phys. Lett. B 276, 127 (1992).

[3] R. A. Porto and A. Zee, Phys. Lett. B 666, 491 (2008).

[4] Y. BenTov and A. Zee, Int. J. Mod. Phys. A 28, 1350149 (2013).

[5] C. T. Hill, Phys. Rev. D 89, 073003 (2014).

[6] V. A. Miransky, M. Tanabashi, and K. Yamawaki, Mod. Phys. Lett. A 04, 1043 (1989).

[7] W. A. Bardeen, C. T. Hill, and M. Lindner, Phys. Rev. D 41, 1647 (1990).

[8] C. T. Hill and E. H. Simmons, Phys. Rep. 381, 235 (2003); 390, 553(E) (2004).

[9] D. F. Litim and F. Sannino, J. High Energy Phys. 12 (2014) 178.

[10] A. D. Bond, G. Hiller, K. Kowalska, and D. F. Litim, J. High Energy Phys. 08 (2017) 004.

[11] S. Abel, E. Mølgaard, and F. Sannino, Phys. Rev. D 99, 035030 (2019).

[12] C. T. Hill, P. A. Machado, A. E. Thomsen, and J. Turner, arXiv:1904.04257.

[13] G. Barenboim, Phys. Rev. D 82, 093014 (2010).

[14] G. Dvali and L. Funcke, Phys. Rev. D 93, 113002 (2016).

[15] L. Randall and R. Sundrum, Phys. Rev. Lett. 83, 4690 (1999).

[16] N. Arkani-Hamed, H. Georgi, and M. D. Schwartz, Ann. Phys. (Amsterdam) 305, 96 (2003).

[17] C. de Rham and G. Gabadadze, Phys. Rev. D 82, 044020 (2010).

[18] Y. Nambu and G. Jona-Lasinio, Phys. Rev. 122, 345 (1961).

[19] H. Georgi and S. L. Glashow, Phys. Rev. Lett. 32, 438 (1974).

[20] B. Pendleton and G. G. Ross, Phys. Lett. 98B, 291 (1981).

[21] C. T. Hill, Phys. Rev. D 24, 691 (1981).

[22] B. A. Dobrescu and P. J. Fox, Eur. Phys. J. C 70, 263 (2010).

[23] S. L. Glashow and S. Weinberg, Phys. Rev. D 15, 1958 (1977).

[24] G. Isidori, in Proceedings, 2012 European School of HighEnergy Physics (ESHEP 2012): La Pommeraye, Anjou, France, 2012 (CERN, Geneva, 2014), pp. 69-105.

[25] A. Alloul, N. D. Christensen, C. Degrande, C. Duhr, and B. Fuks, Comput. Phys. Commun. 185, 2250 (2014).

[26] J. Alwall, R. Frederix, S. Frixione, V. Hirschi, F. Maltoni, O. Mattelaer, H. S. Shao, T. Stelzer, P. Torrielli, and M. Zaro, J. High Energy Phys. 07 (2014) 079.

[27] R. D. Ball et al., Nucl. Phys. B867, 244 (2013).

[28] M. Aaboud et al. (ATLAS Collaboration), Phys. Rev. D 98, 032016 (2018).
[29] E. Maguire, L. Heinrich, and G. Watt, J. Phys. Conf. Ser. 898, 102006 (2017).

[30] R. V. Harlander, Eur. Phys. J. C 76, 252 (2016).

[31] ATLAS Collaboration, Report No. ATLAS-CONF-20170292017.

[32] A. Crivellin, A. Kokulu, and C. Greub, Phys. Rev. D 87, 094031 (2013).

[33] Y. Amhis et al. (Heavy Flavor Averaging Group), arXiv: 1412.7515.

[34] J. Brod and M. Gorbahn, Phys. Rev. Lett. 108, 121801 (2012).

[35] A. Lenz, in Proceedings of the 2012 Electroweak Interactions and Unified Theories: Proceedings of the 47th Rencontres de Moriond on Electroweak Interactions and Unified Theories, La Thuile, 2012, [arXiv:1205.1444].

[36] A. Gando et al. (KamLAND Collaboration), Phys. Rev. D 88, 033001 (2013).

[37] K. Abe et al. (Super-Kamiokande Collaboration), Phys. Rev. D 94, 052010 (2016).

[38] K. Abe et al. (Super-Kamiokande Collaboration), Phys. Rev. D 97, 072001 (2018).

[39] P. Adamson et al. (NOvA Collaboration), Phys. Rev. Lett. 118, 151802 (2017).

[40] K. Abe et al. (T2K Collaboration), Phys. Rev. Lett. 118, 151801 (2017).

[41] P. Adamson et al. (NOvA Collaboration), Phys. Rev. Lett. 118, 231801 (2017).

[42] K. Abe et al. (T2K Collaboration), Phys. Rev. D 96, 092006 (2017); 98, 019902(E) (2018).

[43] N. Aghanim et al. (Planck Collaboration), arXiv:1807 .06209 .

[44] P. Minkowski, Phys. Lett. 67B, 421 (1977).

[45] R. N. Mohapatra and J. W. F. Valle, Phys. Rev. D 34, 1642 (1986).

[46] M. Gell-Mann, P. Ramond, and R. Slansky, Conf. Proc. C 790927, 315 (1979).

[47] R. N. Mohapatra and G. Senjanovic, Phys. Rev. Lett. 44, 912 (1980).

[48] R. N. Mohapatra and G. Senjanovic, Phys. Rev. D 23, 165 (1981).

[49] T. Yanagida, Conf. Proc. C 7902131, 95 (1979).

[50] J. Schechter and J. W. F. Valle, Phys. Rev. D 22, 2227 (1980).

[51] G. Lazarides, Q. Shafi, and C. Wetterich, Nucl. Phys. B181, 287 (1981).

[52] A. de Gouvêa and A. Kobach, Phys. Rev. D 93, 033005 (2016). 
[53] U. Bellgardt et al. (SINDRUM Collaboration), Nucl. Phys. B299, 1 (1988).

[54] K. Hayasaka et al., Phys. Lett. B 687, 139 (2010).

[55] K. Hayasaka et al. (Belle Collaboration), Phys. Lett. B 666 , 16 (2008).

[56] A. M. Baldini et al. (MEG Collaboration), Eur. Phys. J. C 76, 434 (2016).

[57] M. Tanabashi et al. (Particle Data Group), Phys. Rev. D 98, 030001 (2018).
[58] I. Esteban, M. C. Gonzalez-Garcia, M. Maltoni, I. MartinezSoler, and T. Schwetz, J. High Energy Phys. 01 (2017) 087.

[59] P. F. Harrison, D. H. Perkins, and W. G. Scott, Phys. Lett. B 530, 167 (2002).

[60] Z.-z. Xing, Phys. Lett. B 533, 85 (2002).

[61] P. F. Harrison and W. G. Scott, Phys. Lett. B 535, 163 (2002).

[62] X. G. He and A. Zee, Phys. Lett. B 560, 87 (2003). 